\title{
Spin-dependent boundary conditions for isotropic superconducting Green's functions
}

\author{
Audrey Cottet,,${ }^{1,2}$ Daniel Huertas-Hernando, ${ }^{3}$ Wolfgang Belzig, ${ }^{4}$ and Yuli V. Nazarov ${ }^{5}$ \\ ${ }^{1}$ Ecole Normale Supérieure, Laboratoire Pierre Aigrain, 24 rue Lhomond, F-75231 Paris Cedex 05, France \\ ${ }^{2}$ CNRS UMR 8551, Laboratoire associé aux Universités Pierre et Marie Curie et Denis Diderot, France \\ ${ }^{3}$ Department of Physics, Norwegian University of Science and Technology, N-7491 Trondheim, Norway \\ ${ }^{4}$ Department of Physics, University of Konstanz, D-78457 Konstanz, Germany \\ ${ }^{5}$ Kavli Institute of NanoScience, Delft University of Technology, NL-2628 CJ Delft, The Netherlands \\ (Received 23 July 2009; revised manuscript received 27 October 2009; published 23 November 2009)
}

\begin{abstract}
The quasiclassical theory of superconductivity provides the most successful description of diffusive heterostructures comprising superconducting elements, namely, the Usadel equations for isotropic Green's functions. Since the quasiclassical and isotropic approximations break down close to interfaces, the Usadel equations have to be supplemented with boundary conditions for isotropic Green's functions (BCIGF), which are not derivable within the quasiclassical description. For a long time, the BCIGF were available only for spindegenerate tunnel contacts, which posed a serious limitation on the applicability of the Usadel description to modern structures containing ferromagnetic elements. In this paper, we close this gap and derive spindependent BCIGF for a contact encompassing superconducting and ferromagnetic correlations. This finally justifies several simplified versions of the spin-dependent BCIGF, which have been used in the literature so far. In the general case, our BCIGF are valid as soon as the quasiclassical isotropic approximation can be performed. However, their use requires the knowledge of the full scattering matrix of the contact, an information usually not available for realistic interfaces. In the case of a weakly polarized tunnel interface, the BCIGF can be expressed in terms of a few parameters, i.e., the tunnel conductance of the interface and five conductancelike parameters accounting for the spin dependence of the interface scattering amplitudes. In the case of a contact with a ferromagnetic insulator, it is possible to find explicit BCIGF also for stronger polarizations. The BCIGF derived in this paper are sufficiently general to describe a variety of physical situations and may serve as a basis for modeling realistic nanostructures.
\end{abstract}

DOI: 10.1103/PhysRevB.80.184511

PACS number(s): 73.23. $-\mathrm{b}, 74.45 .+\mathrm{c}, 85.75 .-\mathrm{d}$

\section{INTRODUCTION}

The quantum-mechanical spin degree of freedom is widely exploited to control current transport in electronic circuits nowadays. For instance, the readout of magnetic hard disks is based on the giant magnetoresistance effect, which provides the possibility to tune the conductance of, e.g., a ferromagnet/normal-metal/ferromagnet $(F / N / F)$ trilayer by changing the magnetizations of the two $F$ layers from a parallel to an antiparallel configuration. ${ }^{1}$ However, many functionalities of hybrid circuits enclosing ferromagnetic elements remain to be explored. Presently, noncollinear spin transport is triggering an intense activity due to spincurrent-induced magnetization torques, ${ }^{2}$ which offer new possibilities to build nonvolatile memories. ${ }^{3}$ Another interesting possibility is to include superconducting elements in hybrid circuits. When a $N$ layer is connected to a BardeenCooper-Schrieffer (BCS) superconductor $(S)$, the singlet electronic correlations characteristic of $S$ can propagate into $N$ because electrons and holes with opposite spins are coupled coherently by Andreev reflections occurring at the $S / N$ interface. ${ }^{4}$ This so-called "superconducting proximity effect" is among other responsible for strong modifications in the density of states of $N .{ }^{5}$ In a ferromagnet $(F)$, the ferromagnetic exchange field $E_{e x}$, which breaks the symmetry between the two spin bands, is antagonistic to the BCS-type singlet superconducting order. However, this does not exclude the superconducting proximity effect. First, when the magnetization direction is uniform in a whole $S / F$ circuit, superconducting correlations can occur between electrons and holes from opposite-spin bands, like in the $S / N$ limit. These correlations propagate on a characteristic distance limited by the ferromagnetic coherence length $\sqrt{\hbar D / E_{e x}}$, where $D$ is the diffusion coefficient. Furthermore, $E_{e x}$ produces an energy shift between the correlated electrons and holes in the opposite-spin bands, which leads to spatial oscillations of the superconducting order parameter in $F,{ }^{6}$ as recently observed. ${ }^{7-9}$ These oscillations allow to build new types of electronic devices, such as Josephson junctions with negative critical currents, ${ }^{10}$ which promise applications in the field of superconducting circuits. ${ }^{11,12}$ Second, when the circuit encloses several ferromagnetic elements with noncollinear magnetizations, spin-precession effects allow the existence of superconducting correlations between equal spins. ${ }^{13}$ These correlations are expected to propagate in a $F$ on a distance much longer than opposite-spin correlations. This property could be used, e.g., to engineer a magnetically switchable Josephson junction. These and many more effects have been reviewed recently..$^{14,15}$

To model the behavior of superconducting hybrid circuits, a proper description of the interfaces between the different materials is crucial. This paper focuses on the so-called diffusive limit, which is appropriate for most nanostructures available nowadays. In this limit, a nanostructure can be separated into interfaces (or contacts) and regions characterized by isotropic Green's functions $\check{G}$, which do not depend on the direction of the momentum but conserve a possible dependence on spatial coordinates. The spatial evolution of 
the isotropic Green's functions $\check{G}$ is described by Usadel equations. ${ }^{16}$ One needs boundary conditions to relate the values of $\check{G}$ at both sides of an interface. For a long time, the only boundary conditions for isotropic Green's functions (BCIGF) available were spin-independent BCIGF derived for a $S / N$ tunnel contact. ${ }^{17}$ The only interfacial parameter involved in these BCIGF was the tunnel conductance $G_{T}$ of the contact. Such a description is incomplete for a general diffusive spin-dependent interface. Spin-dependent $S / F$ boundary conditions have been first introduced in the ballistic regime. ${ }^{18-21}$ Recently, many references have used spindependent BCIGF (Refs. 22-32) to study the behavior of hybrid circuits enclosing BCS superconductors, ferromagnetic insulators, ferromagnets, and normal metals. These BCIGF, which have been first introduced in Ref. 22, include the $G_{T}$ term of Ref. 17. They furthermore take into account the spin polarization of the interface tunnel probabilities through a $G_{M R}$ term, and the spin dependence of interfacial scattering phase shifts through $G_{\phi}$ terms. It has been shown that the $G_{M R}$ and $G_{\phi}$ terms lead to a rich variety of effects. First, the $G_{\phi}$ terms can produce effective Zeeman fields inside thin superconducting or normal-metal layers, ${ }^{22-24}$ an effect which could be used, e.g., to implement an absolute spin valve. ${ }^{22}$ In thick superconducting layers, this effect is replaced by spin-dependent resonances occurring at the edges of the layers. ${ }^{25}$ Second, the $G_{\phi}$ terms can shift the spatial oscillations of the superconducting order parameter in ferromagnets. ${ }^{24-26}$ Third, the $G_{\phi}$ term can produce superconducting correlations between equal spins, e.g., in a circuit enclosing a BCS superconductor and several ferromagnetic insulators magnetized in noncollinear directions. ${ }^{30}$ The $G_{M R}$ terms have been taken into account for a chaotic cavity connected to a superconductor and several ferromagnets. ${ }^{28,29}$ In this system, crossed Andreev reflections and direct electron transfers are responsible for nonlocal transport properties. The ratio between these two kinds of processes, which determines, e.g., the sign of the nonlocal conductance, ${ }^{33,34}$ can be controlled through the relative orientation of the ferromagnets magnetizations.

In this paper, we present a detailed derivation of the spindependent BCIGF based on a scattering description of interfaces. Our results thus provide a microscopic basis for all future investigations of ferromagnet-superconductor diffusive heterostructures taking into account the spin-dependent interface scattering. To make the BCIGF comprehensive and of practical value, we make a series of sequential assumptions, starting from very general to more and more restrictive hypotheses. In a first part, we assume that the contact is fully metallic, i.e., it connects two conductors which can be superconductors, ferromagnets, or normal metals. We consider ferromagnets with exchange fields much smaller than their Fermi energies, as required for the applicability of the quasiclassical isotropic description. We assume that the contact nevertheless produces a spin-dependent scattering due to a spin-dependent interfacial barrier $\bar{V}_{b}$. In this case, we establish general BCIGF which require the knowledge of the full contact scattering matrix. Then, we assume that the contact locally conserves the transverse channel index (specular hypothesis) and spins collinear to the contact magnetization. In the tunnel limit, assuming $\bar{V}_{b}$ is weakly spin dependent, we find that the BCIGF involve the $G_{T}, G_{M R}$, and $G_{\phi}$ terms used in Refs. 24-32, plus additional $G_{\chi}$ terms which are usually disregarded. In a second part, we study a specular contact connecting a metal to a ferromagnetic insulator $(F I)$. If we assume a weakly spin-dependent interface scattering, we obtain the BCIGF used in Refs. 22 and 23. We also present BCIGF valid beyond this approximation. Note that the various BCIGF presented in this paper can be applied to noncollinear geometries.

Most of the literature on superconducting hybrid circuits uses a spatially continuous description, i.e., in each conductor, the spatial dependence of the Green's function $\check{G}$ is explicitly taken into account. The BCIGF presented in this paper can also be used in the alternative approach of the socalled circuit theory. This approach is a systematic method to describe multiterminal hybrid structures, in order to calculate average transport properties ${ }^{35-37}$ but also current statistics. ${ }^{38,39}$ It relies on the mapping of a real geometry onto a topologically equivalent circuit represented by finite elements. The circuit is split up into reservoirs (voltage sources), connectors (contacts, interfaces), and nodes (small electrodes) in analogy to classical electric circuits. Each reservoir or node is characterized by an isotropic Green's function $\breve{G}$ without spatial dependence, which plays the role of a generalized potential. One can define matrix currents, which contain information on the flows of charge, spin, and electron/hole coherence in the circuit. Circuit theory requires that the sum of all matrix currents flowing from the connectors into a node is balanced by a "leakage" current which accounts for the nonconservation of electron/hole coherence and spin currents in the node. This can be seen as a generalized Kirchhoff's rule and completely determines all the properties of the circuit. So far, circuit theory has been developed separately for $F / N$ (Ref. 35) and $S / N$ circuits (Ref. 37). Throughout this paper, we express the BCIGF in terms of matrix currents. Our work thus allows a straightforward generalization of circuit theory to the case of multiterminal circuits which enclose superconductors, normal metals, ferromagnets, and ferromagnetic insulators, in a possibly noncollinear geometry.

This paper is organized as follows. We first consider the case of a metallic contact, i.e., a contact between two conductors. Section II defines the general and isotropic Green's functions $G$ and $\check{G}$ used in the standard description of hybrid circuits encompassing BCS superconductors. Section III introduces the ballistic Green's function $\widetilde{g}$, which we use in our derivation. Section IV discusses the scattering description of the contact with a transfer matrix $\bar{M}$. Although we consider the diffusive limit, the scattering description is relevant for distances to the contact shorter than the elastic mean-free path. On this scale, one can use $\bar{M}$ to relate the left and right ballistic Green's functions $\widetilde{g}_{L}$ and $\widetilde{g}_{R}$. Section V presents an isotropization scheme which accounts for impurity scattering and leads to the isotropic Green's functions $\check{G}_{L(R)}$ away from the contact. Section VI establishes the general metallic BCIGF which relate $\check{G}_{L}, \check{G}_{R}$, and $\bar{M}$. Section VII gives more transparent expressions of these BCIGF in various limits. 


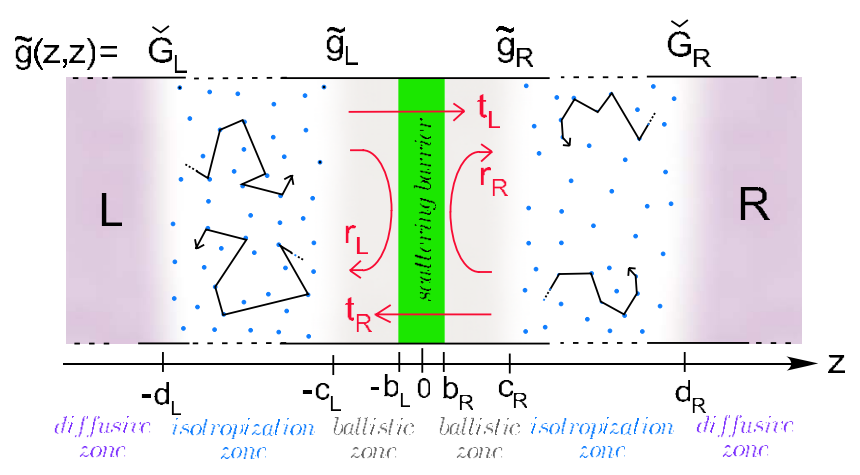

FIG. 1. (Color online) Model of a planar contact between two diffusive conductors $L$ and $R$. The inner part of the model consists of two ballistic zones (light gray areas), connected together by a scattering barrier $V_{b}(z)$ located at $z \in\left[-b_{L}, b_{R}\right]$ (dark gray area, green online). We set the end of the left/right ballistic zone at $z$ $=\mp c_{L(R)}$ with $c_{L(R)}-b_{L(R)}$ a distance smaller or equal to the elastic mean-free path $\ell_{\mathrm{e}}^{L(R)}$ of $L(R)$. At this place, the ballistic Green's function $\widetilde{g}(z, z, \varepsilon)$ equals $\widetilde{g}_{L(R)}$. The effect of $V_{b}(z)$ can be described with electronic-transmission amplitudes $t_{L(R)}$ and reflection amplitudes $r_{L(R)}$. There exists a simple relation between $\widetilde{g}_{R}$ and $\widetilde{g}_{L}$, based on the scattering parameters $t_{L(R)}$ and $r_{L(R)}$ [see Eq. (22)]. The two ballistic zones are connected to two isotropization zones which have a size $d_{L(R)}$ of the order of a few $\ell_{\mathrm{e}}^{L(R)}$ (dotted areas). In the isotropization zones, the electron dynamics is dominated by elasticimpurity scattering. Hence, $\widetilde{g}(z, z, \varepsilon)$ becomes isotropic away from the contact region, and one can define, beyond the isotropization zones, two diffusive zones (gray areas, purple online) described by the isotropic Green's function $\check{G}(z, \varepsilon)$. For $z \rightarrow \mp d_{L(R)}$, $\widetilde{g}(z, z, \varepsilon)$ tends to $\check{G}(z, \varepsilon)=\check{G}_{L(R)}$. Note that since the transition between the ballistic, isotropization, and diffusive zones is smooth, the choice of the coordinates $c_{L(R)}$ and $d_{L(R)}$ in Fig. 1 is somewhat arbitrary, i.e., defined only up to an uncertainty of the order of a fraction of $\ell_{\mathrm{e}}^{L(R)}$ or $\ell_{\mathrm{e}}^{L(R)}$, respectively. However, this choice does not affect the BCIGF as we show in the text.

Section VIII addresses the case of a contact with a FI side, in analogy with the treatment realized in the metallic case. Section IX concludes. Appendix A discusses the structure of the transfer matrix $\bar{M}$ and Appendix B gives details on the calculation of the matrix current. Appendix $\mathrm{C}$ relates our BCIGF to the equations previously obtained in the normalstate limit. ${ }^{35,36}$ Appendix D discusses the BCIGF obeyed by the retarded parts of $\check{G}_{L(R)}$ in the collinear case. For completeness, Appendix E presents the Usadel equations in our conventions.

\section{GENERAL AND ISOTROPIC GREEN'S FUNCTIONS}

In Secs. II-VII, we consider a planar metallic contact between two diffusive conductors noted $L$ (left conductor) and $R$ (right conductor) (see Fig. 1). The conductor $L[R]$ can exhibit spin and/or superconducting correlations due to its superconducting order parameter $\Delta$ or exchange field $E_{e x}$, or due to the proximity effect with other conductors. For the primary description of electronic correlations in $L$ and $R$, one can use a general Green's function $G$ defined in the Keldysh $\otimes$ Nambu $\otimes$ Spin $\otimes$ Coordinate space. In the stationary case, $\mathrm{G}$ can be defined as

$$
\mathrm{G}\left(\vec{r}, \vec{r}^{\prime}, \varepsilon\right)=\int \frac{d t}{\hbar} \mathrm{G}\left(\vec{r}, \vec{r}^{\prime}, t-t^{\prime}\right) \exp \left\{i \varepsilon \frac{t-t^{\prime}}{\hbar}\right\}
$$

with

$$
\begin{aligned}
G\left(\vec{r}, \vec{r}^{\prime}, t-t^{\prime}\right) & =\left[\begin{array}{cc}
G^{r}\left(\vec{r}, \vec{r}^{\prime}, t-t^{\prime}\right) & G^{K}\left(\vec{r}, \vec{r}^{\prime}, t-t^{\prime}\right) \\
0 & \mathbb{G}^{a}\left(\vec{r}, \vec{r}^{\prime}, t-t^{\prime}\right)
\end{array}\right], \\
G^{r(a)}\left(\vec{r}, \vec{r}^{\prime}, t-t^{\prime}\right) & =\mp i \theta\left[ \pm\left(t-t^{\prime}\right)\right] \check{\tau}_{3}\left\langle\left\{\Psi(t, \vec{r}), \Psi^{\dagger}\left(t^{\prime}, \vec{r}^{\prime}\right)\right\}\right\rangle
\end{aligned}
$$

and

$$
\mathbb{G}^{K}\left(\vec{r}, \vec{r}^{\prime}, t-t^{\prime}\right)=-i \check{\tau}_{3}\left\langle\left[\boldsymbol{\Psi}(t, \vec{r}), \boldsymbol{\Psi}^{\dagger}\left(t^{\prime}, \vec{r}^{\prime}\right)\right]\right\rangle .
$$

Here, $[\cdots, \cdots]$ and $\{\cdots, \cdots\}$ denote commutators and anticommutators, respectively, $\vec{r}$ and $\vec{r}^{\prime}$ are space coordinates, $t$ and $t^{\prime}$ are time coordinates, and $\varepsilon$ is the energy. We use a spinor representation of the fermion operators, i.e.,

$$
\boldsymbol{\Psi}^{\dagger}(t, \vec{r})=\left[\Psi_{\uparrow}^{\dagger}(t, \vec{r}),-\Psi_{\downarrow}^{\dagger}(t, \vec{r}), \Psi_{\uparrow}(t, \vec{r}), \Psi_{\downarrow}(t, \vec{r})\right]
$$

in the Nambu $\otimes$ Spin space. We denote by $\check{\tau}_{3}$ the third Nambu-Pauli matrix, i.e., $\check{\tau}_{3}=\operatorname{diag}(1,1,-1,-1)$ in the $\mathrm{Nambu} \otimes$ Spin space. For later use, we also define the third spin Pauli matrix, i.e., $\check{\sigma}_{Z}=\operatorname{diag}(1,-1,1,-1)$. With the above conventions, the Green's function $G$ follows the Gorkov equations

$$
\left[\varepsilon \check{\tau}_{3}-H(\vec{r})+i \check{\Delta}(z)-\check{\Sigma}_{i m p}(z)\right] \mathrm{G}\left(\vec{r}, \vec{r}^{\prime}, \varepsilon\right)=\delta\left(\vec{r}, \vec{r}^{\prime}\right)
$$

and

$$
\mathrm{G}\left(\vec{r}, \vec{r}^{\prime}, \varepsilon\right)\left[\varepsilon \check{\tau}_{3}-H\left(\vec{r}^{\prime}\right)+i \check{\Delta}\left(z^{\prime}\right)-\check{\Sigma}_{\text {imp }}\left(z^{\prime}\right)\right]=\delta\left(\vec{r}, \vec{r}^{\prime}\right)
$$

Here, $\check{\Delta}$ corresponds to the gap matrix associated to a BCS superconductor (see definition in Appendix E). Hamiltonian $H(\vec{r})$ can be decomposed as

$$
H(\vec{r})=H_{l}(z)+H_{t}(\vec{\rho})+\bar{V}_{b}(z, \vec{\rho})
$$

where $z$ and $\vec{\rho}$ are the longitudinal and transverse components of $\vec{r}$. The part $H_{l}(z)=-\left(\hbar^{2} / 2 m\right) \partial^{2} / \partial_{z}^{2}-E_{e x}(z) \check{\sigma}_{Z}-E_{F}(z)$ includes a ferromagnetic exchange field $E_{e x}(z)$ in the direction $\vec{Z}$ and the Fermi energy $E_{F}(z \lessgtr 0)=E_{F, L(R)}$, whereas the part $H_{t}(\vec{\rho})=-\left(\hbar^{2} / 2 m\right) \partial^{2} / \partial_{\vec{\rho}}^{2}+V_{c}(\vec{\rho})$ includes a lateral confinement potential $V_{c}(\vec{\rho})$. The potential barrier $\bar{V}_{b}(z, \vec{\rho})$ describes a possibly spin-dependent and nonspecular interface. It is finite in the area $z \in\left[-b_{L}, b_{R}\right]$ only. In the Born approximation, the impurity self-energy at side $Q \in\{L, R\}$ of the interface can be expressed as $\check{\Sigma}_{i m p}(z, \varepsilon)=-i \hbar \check{G}(z, \varepsilon) / 2 \tau_{Q}$. Here, the impurity elastic-scattering time $\tau_{Q}$ in material $Q$ can be considered as spin independent due to $E_{e x} \ll E_{F}$. The Green's function $\check{G}(z, \varepsilon)$, which has already been mentioned in Sec. I, corresponds to the quasiclassical and isotropic average of $G$ inside conductor $L(R)$. It can be calculated as ${ }^{40}$

$$
\check{G}(z, \varepsilon)=i \mathrm{G}\left(\vec{r}=\vec{R}, \vec{r}^{\prime}=\vec{R}, \varepsilon\right) / \pi \nu_{0}
$$

with $z$ the longitudinal component of $\vec{R}$ and $\nu_{0}$ the density of states per spin direction and unit volume for free electrons. 
TABLE I. Meaning of the various decorations used in this paper for operators defined in the $\mathcal{E}$ space. Each decoration corresponds to a particular structure in the transverse channels (index $n$ ), propagation direction (index $s$ ), spin (index $\sigma$ ), Nambu (index $\nu$ ) and Keldysh subspaces.

\begin{tabular}{cccccc}
\hline \hline \multicolumn{5}{c}{ Structure allowed in the subspaces } \\
\cline { 2 - 6 } & Channels $n$ & Direction $s$ & Spins $\sigma$ & Nambu $\nu$ & Keldysh \\
\hline$\tilde{A}$ & Yes & Yes & Yes & Yes & Yes \\
$\check{A}$ & No & No & Yes & Yes & Yes \\
$\check{A}$ & Yes & Yes & Yes & Diagonal & No \\
$\bar{A}$ & Yes & Yes & Yes & No & No \\
$\hat{A}$ & Yes & Yes & No & No & No \\
$\bar{A}$ & Yes & No & No & No & No \\
\hline \hline
\end{tabular}

Since we consider the case of wide contacts, $\check{G}, \check{\Sigma}_{\text {imp }}$, and $\check{\Delta}$ can be considered as independent from $\vec{\rho}$.

In this paper, we consider the diffusive (i.e., quasiclassical and isotropic) limit, i.e.,

$$
E_{e x},|\Delta|, \varepsilon, k_{B} T \ll \hbar / \tau_{Q} \ll E_{F},
$$

where $T$ is the temperature and $k_{B}$ is the Boltzmann constant. In this regime, the spatial evolution of $\breve{G}(z, \varepsilon)$ inside $L$ and $R$ is described by the Usadel equations which follow from Eqs. (6) and (7) (see Appendix E). The characteristic distances occurring in the Usadel equations are $\xi_{F}=\left(\hbar D_{F} / E_{e x}\right)^{1 / 2}, \xi_{N}$ $=\left(\hbar D_{N} / 2 k_{B} T\right)^{1 / 2}$, and $\xi_{S}=\left(\hbar D_{S} / 2 \Delta\right)^{1 / 2}$ for a ferromagnet $F$, a normal metal $N$ and a superconductor $S$, respectively, with $D_{Q}=v_{F, Q}^{2} \tau_{Q} / 3$ and $v_{F, Q}$ the diffusion constant and Fermi velocities of material $Q$. According to Eq. (10), the scale $\xi_{Q}$ is much larger than the elastic mean-free path $\ell_{\mathrm{e}}^{Q}=v_{F, Q} \tau_{Q}$. Importantly, the Usadel equations alone are not sufficient to describe the behavior of diffusive hybrid circuits. One also needs to relate the values of $\breve{G}$ at both sides of an $L / R$ interface with BCIGF, which we derive in the next sections.

For the sake of concreteness, we give typical order of magnitudes for the different length scales involved in the problem. These length scales strongly depend on the detailed composition and structure of the materials and interfaces considered so that the applicability of the quasiclassical isotropic description has to be checked in each case. The value of $b_{L(R)}$ can strongly vary from a few atomic layers to a few nanometers if the two materials constituting the interface interdiffuse. ${ }^{41}$ The mean-free path, which strongly depends on the impurity concentration, can be of the order of a few nanometers. ${ }^{8}$ The superconducting lengthscale $\xi_{S}$ is usually of the order of $10 \mathrm{~nm}$ for Niobium. ${ }^{42,43}$ The Cooper-pair penetration length can reach $\xi_{F} \sim 10 \mathrm{~nm}$ for a diluted magnetic allow such as CuNi (Ref. 8) or $\xi_{N} \sim 1000 \mathrm{~nm}$ for a normal metal such as $\mathrm{Cu}$ at $T=20 \mathrm{mK}{ }^{44}$

It is worth to note, at this point, that the derivation presented below is not restricted to stationary problems on superconducting heterostructures. Actually most of the derivations made below do not rely on the specific Keldysh structure introduced in Eqs. (1)-(4) and our results can be directly used to describe full counting statistics in the ex- tended Keldysh technique ${ }^{39}$ or multiple Andreev reflections. ${ }^{45}$ In fact, boundary conditions for arbitrary timedependent scattering problems have been recently formulated in a similar spirit. ${ }^{46}$ However, having in mind the many concrete applications of the boundary conditions in superconducting heterostructures and keeping the notation as simple as possible, we derive the BCIGF below in the framework of the stationary Keldysh-Nambu Green's functions.

\section{BALLISTIC GREEN'S FUNCTION}

Considering the structure of Eqs. (6)-(8), for $z, z^{\prime}<-b_{L}$ or $z, z^{\prime}>b_{R}$, one can expand $\mathrm{G}$ in transverse modes as ${ }^{47}$

$$
\begin{aligned}
& \mathbb{G}^{\nu \sigma, \nu^{\prime} \sigma^{\prime}}\left(\vec{r}, \vec{r}^{\prime}, \varepsilon\right) \\
& =\sum_{n s, n^{\prime} s^{\prime}}\left(\widetilde{\mathcal{G}}_{n s, n^{\prime} s^{\prime}}^{v \sigma, \sigma^{\prime}}\left(z, z^{\prime}, \varepsilon\right) \frac{\chi_{n}(\vec{\rho}) \chi_{m}^{*}\left(\vec{\rho}^{\prime}\right)}{2 \pi \hbar \sqrt{v_{n}(z, \varepsilon) v_{m}\left(z^{\prime}, \varepsilon\right)}}\right. \\
& \left.\quad \quad \times \exp \left[i s k_{n}(z) z-i s^{\prime} k_{m}\left(z^{\prime}\right) z^{\prime}\right]\right)
\end{aligned}
$$

In this section, we use spin indices $\sigma, \sigma^{\prime} \in\{\uparrow, \downarrow\}$ which correspond to spin directions parallel or antiparallel to the direction $\vec{Z}$, and Nambu indices $\nu, \nu^{\prime} \in\{e, h\}$ for electron and hole states. The indices $s, s^{\prime} \in\{+,-\}$ account for the longitudinal direction of propagation (we use $\sigma, \sigma^{\prime}= \pm 1, \nu, \nu^{\prime}$ $= \pm 1$, and $s, s^{\prime}= \pm 1$ in mathematical expressions). We introduce the wave function $\chi_{n}(\vec{\rho})$ for the transverse channel $n$, i.e., $H_{t}(\vec{\rho}) \chi_{n}(\vec{\rho})=E_{n} \chi_{n}(\vec{\rho})$, and the corresponding longitudinal momentum and velocity, i.e., $k_{n}(z)=\left\{2 m\left[E_{F}(z)-E_{n}\right]\right\}^{1 / 2} / \hbar$ and $v_{n}(z)=\hbar k_{n}(z) / m$. Importantly, we have disregarded the dependences of $k_{n}$ and $v_{n}$ on $E_{e x}$ and $\varepsilon$ due to Eq. (10). The $\sim$ decoration denotes that the Green's function $\tilde{\mathcal{G}}$ can have a general structure in the Keldysh $\otimes \mathrm{Nambu} \otimes \mathrm{Spin} \otimes$ Channel $\otimes$ Direction space, noted $\mathcal{E}$ in the following. In contrast, $\checkmark$ denotes the fact that $\check{G}_{L(R)}$ has no structure in the Channel $\otimes$ Direction subspace, noted $\mathcal{E}$ in the following (see the summary of notations in Table I). Due to Eqs. (6) and (7), $\widetilde{\mathcal{G}}\left(z, z^{\prime}, \varepsilon\right)$ is not continuous at $z=z^{\prime} \cdot{ }^{37,47}$ One can use ${ }^{48,49}$ 


$$
\widetilde{\mathcal{G}}\left(z, z^{\prime}, \varepsilon\right)=-i \pi\left[\widetilde{g}\left(z, z^{\prime}, \varepsilon\right)+\hat{\Sigma}_{3} \operatorname{sgn}\left(z-z^{\prime}\right)\right]
$$

with $\hat{\Sigma}_{3}$ as the third Pauli matrix in the direction of propagation space, i.e., $\left(\hat{\Sigma}_{3}\right)_{n s, m s^{\prime}}^{\sigma, \sigma^{\prime}}=s \delta_{s s^{\prime}} \delta_{n m} \delta_{\sigma \sigma^{\prime}} \delta_{\nu \nu^{\prime}} \mathbf{1}_{K}$. Equation (12) involves a ballistic Green's function $\widetilde{g}\left(z, z^{\prime}, \varepsilon\right) \in \mathcal{E}$ which is continuous at $z=z^{\prime}$. We will see below that this quantity plays a major role in the derivation of the BCIGF.

For later use, we now derive the equations of evolution followed by $\widetilde{\mathcal{G}}$. Inserting Eq. (11) into Eqs. (6) and (7), one can check that, for $z \neq z^{\prime}$ and $z, z^{\prime}<-b_{L}(Q=L)$ or $z, z^{\prime}$ $>b_{R}(Q=R), \tilde{\mathcal{G}}$ follows the equations

$$
\left[i \hbar \hat{\Sigma}_{3} \overline{\bar{v}}_{Q} \frac{\partial}{\partial z}+i \check{\Delta}-\check{\Sigma}_{i m p}(z)\right] \otimes \widetilde{\mathcal{G}}\left(z, z^{\prime}, \varepsilon\right)=0
$$

and

$$
\widetilde{\mathcal{G}}\left(z, z^{\prime}, \varepsilon\right) \otimes\left[-i \hbar \hat{\Sigma}_{3} \overline{\bar{v}}_{Q} \frac{\partial}{\partial z^{\prime}}+i \check{\Delta}-\check{\Sigma}_{\text {imp }}\left(z^{\prime}\right)\right]=0 .
$$

We have introduced above a velocity matrix $\overline{\bar{v}}_{Q}$ with a structure in the channels subspace only, i.e., $\left(\overline{\bar{v}}_{L(R)}\right)_{n s, n^{\prime} s^{\prime}}^{\nu \sigma, \sigma^{\prime}}=v_{n}(z$ $\lessgtr 0) \delta_{s s^{\prime}} \delta_{n n^{\prime}} \delta_{\sigma \sigma^{\prime}} \delta_{v v^{\prime}} \mathbf{1}_{K}$, with $\mathbf{1}_{K}$ as the identity matrix in the Keldysh space. We have furthermore assumed that the socalled envelope function $\widetilde{\mathcal{G}}$ varies smoothly on the scale of the Fermi wavelength, in order to neglect terms proportional to $\partial^{2} / \partial z^{2}$ and $\partial^{2} / \partial z^{\prime 2}$ in Eqs. (13) and (14). ${ }^{47}$

\section{SCATTERING DESCRIPTION OF A METALLIC CONTACT}

We now define, at both sides of the barrier $\bar{V}_{b}$, two ballistic zones (with no impurity scattering) located at $z \in\left[-c_{L}\right.$, $\left.-b_{L}\right]$ and $z \in\left[c_{R}, b_{R}\right]$, with $c_{L(R)}-b_{L(R)} \leq \ell_{\mathrm{e}}^{L(R)}$ (see Fig. 1). In the region $z \in\left[-c_{L}, c_{R}\right]$, we can disregard the superconducting gap matrix $\check{\Delta}$ since $c_{L}+c_{R} \ll \xi_{S}$. Therefore, the electron and hole dynamics can be described with the Schrödinger equation

$$
\left[\varepsilon \check{\tau}_{3}-H(\vec{r})\right] \phi(\vec{r}, \varepsilon)=0
$$

or, equivalently

$$
\phi^{\dagger}(\vec{r}, \varepsilon)\left[\varepsilon \check{\tau}_{3}-H(\vec{r})\right]=0
$$

whose solution has the form ${ }^{50}$

$$
\phi^{\nu, \sigma}(\vec{r}, \varepsilon)=\sum_{n, s} \psi_{n, s}^{\nu, \sigma}(z, \varepsilon) \frac{\chi_{n}(\vec{\rho})}{\sqrt{2 \pi \hbar v_{n}(z)}} e^{i s \nu k_{n}(z) z}
$$

in the ballistic zones. Here, $\phi(\vec{r}, \varepsilon)$ is a vector in the Spin $\otimes \mathrm{Nambu} \otimes$ Keldysh space, and $\psi(z, \varepsilon)$ is a vector in the $\mathcal{E}$ space. The index $s$ corresponds again to the longitudinal direction of propagation. We have introduced $\nu$ indices in the exponential factors of Eq. (17) because, for the same sign of wave vector, electrons, and holes go in opposite directions. Therefore, in Eq. (17), $s=+/-1$ systematically denotes the right/left-going states. One can introduce a transfer matrix $\mathcal{M}(\varepsilon) \in \mathcal{E}$ such that $\psi\left(c_{R}, \varepsilon\right)=\mathcal{M}(\varepsilon) \psi\left(-c_{L}, \varepsilon\right)$. The matrix
$\breve{\mathcal{M}}$ and the Landauer-Büttiker scattering matrix can be considered as equivalent descriptions of a contact, provided one introduces small but finite transmission coefficients to regularize $\mathcal{M}$ in case of perfectly reflecting channels. This regularization procedure does not affect practical calculations as illustrated in Sec. VII D. Since $H(\vec{r})$ does not couple electron and holes, $\mathcal{M}$ has the structure

$$
\breve{\mathcal{M}}=\left[\begin{array}{cc}
\mathcal{M}^{e}(\varepsilon) & 0 \\
0 & \mathcal{M}^{e}(-\varepsilon)^{*}
\end{array}\right]
$$

in the Nambu subspace. Moreover, $\mathcal{M}$ is proportional to the identity in the Keldysh space, like $H(\vec{r})$. For later use, we point out that flux conservation leads to ${ }^{51}$

$$
\breve{\mathcal{M}}^{\dagger} \hat{\Sigma}_{3} \overline{\mathcal{M}}=\widetilde{\mathcal{M}} \hat{\Sigma}_{3} \breve{\mathcal{M}}^{\dagger}=\hat{\Sigma}_{3} .
$$

We now connect the above scattering approach with the Green's function description. ${ }^{18}$ With the assumptions done in this section, Eqs. (6) and (7) give, for $z \in\left[-c_{L}, c_{R}\right]$ and $z^{\prime}$ $\neq z$

$$
\left[\varepsilon \check{\tau}_{3}-H(\vec{r})\right] \mathrm{G}\left(\vec{r}, \vec{r}^{\prime}, \varepsilon\right)=0
$$

and

$$
\mathrm{G}\left(\vec{r}, \vec{r}^{\prime}, \varepsilon\right)\left[\varepsilon \check{\tau}_{3}-H\left(\vec{r}^{\prime}\right)\right]=0 .
$$

We recall that in the ballistic zones, $G$ takes the form (11). In the domain $z^{\prime}>z$, a comparison between Eqs. (15), (16), (20), and (21) gives, in terms of the decompositions Eqs. (11) and (17)

$$
\tilde{U} \widetilde{\mathcal{G}}\left(c_{R}, c_{R}+0^{-}, \varepsilon\right) \widetilde{U}=\breve{\mathcal{M}}(\varepsilon) \tilde{U} \widetilde{\mathcal{G}}\left(-c_{L}+0^{+},-c_{L}, \varepsilon\right) \widetilde{U} \widetilde{\mathcal{M}}^{\dagger}(\varepsilon) .
$$

We have introduced above the transformation $\widetilde{U}=\left(\mathbf{1}+\check{\tau}_{3}\right.$ $\left.+\left[1-\check{\tau}_{3}\right] \hat{\Sigma}_{1}\right) / 2$ to compensate the fact that the $\nu$ indices do not occur in the exponential terms of Eq. (11). Using Eq. (12), we obtain

$$
\widetilde{g}_{R}=\bar{M} \widetilde{g}_{L} \bar{M}^{\dagger}
$$

with $\widetilde{g}_{L}=\widetilde{g}\left(-c_{L},-c_{L}, \varepsilon\right), \widetilde{g}_{R}=\widetilde{g}\left(c_{R}, c_{R}, \varepsilon\right)$, and

$$
\bar{M}=\left[\begin{array}{cc}
\mathcal{M}^{e}(\varepsilon) & 0 \\
0 & \hat{\Sigma}_{1} \mathcal{M}^{e}(-\varepsilon)^{*} \hat{\Sigma}_{1}
\end{array}\right]
$$

in the Nambu subspace. Note that due to Eq. (19), one has

$$
\bar{M}^{\dagger} \hat{\Sigma}_{3} \bar{M}=\bar{M} \hat{\Sigma}_{3} \bar{M}^{\dagger}=\hat{\Sigma}_{3} \text {. }
$$

We now discuss how spin dependences arise in our problem. Due to the hypotheses required to reach the diffusive limit [see Eq. (10)], we have neglected the dependence of $k_{n}$ and $v_{n}$ on the exchange field $E_{e x}$, and the energy $\varepsilon$. Accordingly, we have to disregard the dependence of $\bar{M}$ on $E_{e x}$ and $\varepsilon$. This does not forbid that $\bar{M}$ depends on spin. Indeed, in the general case, when an interface involves a $F$ material which is ferromagnetic in the bulk, the transfer matrix $\bar{M}$ can 
depend on spin for two reasons: first, the wave vectors of the electrons scattered by the barrier can depend on spin due to $E_{e x}$, and second, the interface barrier potential $\bar{V}_{b}$ can itself be spin dependent. Importantly, one can check that $E_{e x}$ and $\bar{V}_{b}$ occur independently in Eqs. (6) and (7). The value of $E_{e x}$ and the spin dependence of $\bar{V}_{b}$ are not directly related because the second depend on properties such as interfacial disorder or discontinuities in the electronic-band structure, which do not influence $E_{e x}$ far from the interface. Therefore, nothing forbids to have simultaneously $E_{e x} \ll E_{F}$ (this can occur, e.g., in a diluted ferromagnetic alloy such as PdNi) and a spin dependent $\bar{M}$, due to a spin-dependent interface potential $\bar{V}_{b}$. It is even possible to obtain this situation artificially, by fabricating, e.g., a contact with a very thin FI barrier separating two normal metals or superconductors. Note that in spite of $E_{e x} \ll E_{F}$, the exchange field $E_{e x}$ can play a major role in diffusive hybrid circuits by modifying drastically the spatial evolution of the isotropic Green's function $\check{G}_{F}(z, \varepsilon)$ inside a ferromagnetic metal $F$ on the scale $\xi_{F}$ (see Appendix E).

\section{ISOTROPIZATION SCHEME}

In this section, we show that the Green's function $\widetilde{g}\left(z, z^{\prime}=z, \varepsilon\right)$ becomes isotropic in momentum space (i.e., proportional to the identity in the $\mathcal{E}$ subspace) due to impurity scattering, when moving further away from the contact. One can consider that this process occurs in "isotropization zones" with a size $d_{L(R)}$ of the order of a few $\ell_{\mathrm{e}}^{L(R)}$ for side $L(R)$ of the contact ${ }^{52}$ (dotted areas in Fig. 1). Beyond the isotropization zones, quasiparticles reach diffusive zones (see Fig. 1) characterized by isotropic Green's functions $\check{G}(z, \varepsilon)$ with no structure in the $\underline{\mathcal{E}}$ subspace. We show below that $\widetilde{g}\left(z, z^{\prime}=z, \varepsilon\right)$ tends to $\check{G}\left(z=\mp d_{L(R)}, \varepsilon\right)$ at the external borders $z=\mp d_{L(R)}$ of the isotropization zones. Note that the results presented in this section do not depend on the details of the isotropization mechanism.

We study the spatial evolution of $\widetilde{\mathcal{G}}$ in the isotropization zones located at $z \in\left[-d_{L},-c_{L}\right]$ and $z \in\left[c_{R}, d_{R}\right]$, using Eqs. (13) and (14). The superconducting gap matrix $\check{\Delta}$ can be neglected from these equations due to $d_{L(R)} \ll \xi_{S}$. We thus obtain, for the isotropization zone of side $Q$ and $z \neq z^{\prime}$

$$
\left[\hat{\Sigma}_{3} \overline{\bar{v}}_{Q} \frac{\partial}{\partial z}+\frac{\check{G}(z, \varepsilon)}{2 \tau_{Q}}\right] \otimes \widetilde{\mathcal{G}}\left(z, z^{\prime}, \varepsilon\right)=0
$$

and

$$
\widetilde{\mathcal{G}}\left(z, z^{\prime}, \varepsilon\right) \otimes\left[-\hat{\Sigma}_{3} \overline{\bar{v}}_{Q} \frac{\partial}{\partial z^{\prime}}+\frac{\check{G}\left(z^{\prime}, \varepsilon\right)}{2 \tau_{Q}}\right]=0 .
$$

Due to $\xi_{Q} \gg \ell_{\mathrm{e}}^{Q}$, one can disregard the space dependence of $\check{G}(z, \varepsilon)$ in the above equations. We will thus replace $\check{G}(z, \varepsilon)$ by its value $\check{G}_{Q}$ at the beginning of the diffusive zone $Q$, i.e., $\check{G}_{L(R)}=\check{G}\left(z=\mp d_{L(R)}, \varepsilon\right)$. For later use, we recall that $\check{G}_{L}$ and $\check{G}_{R}$ fulfill the normalization condition

$$
\check{G}_{L}^{2}=\check{G}_{R}^{2}=\mathbf{1}
$$

with 1 as the identity in the $\mathcal{E}$ space. In the isotropization zone of side $Q$, Eqs. (12) and (25)-(27) give

$$
\begin{aligned}
\tilde{\mathcal{G}}\left(z, z^{\prime}, \varepsilon\right)= & -i \pi \widetilde{P}_{Q}\left[\lambda_{Q}(z)\right] \\
& \times\left[\tilde{g}_{Q}+\operatorname{sgn}\left(z-z^{\prime}\right) \hat{\Sigma}_{3}\right] \widetilde{P}_{Q}\left[-\lambda_{Q}\left(z^{\prime}\right)\right]
\end{aligned}
$$

with $\lambda_{L(R)}(z)=z \pm c_{L(R)}$ and

$$
\widetilde{P}_{Q}[z]=\operatorname{ch}\left[z / 2 \overline{\bar{v}}_{Q} \tau_{Q}\right]-\hat{\Sigma}_{3} \check{G}_{Q} \operatorname{sh}\left[z / 2 \overline{\bar{v}}_{Q} \tau_{Q}\right]
$$

for $Q \in\{L, R\}$. Note that the choice of the coordinate $d_{L(R)}$ in Fig. 1 is somewhat arbitrary, i.e., defined only up to an uncertainty of the order of $\ell_{\mathrm{e}}^{L(R)}$ because there is a smooth transition between the isotropization and diffusive zones of the contact. As a result, $\widetilde{\mathcal{G}}$ must tend continuously to its limit value $\widetilde{\mathcal{G}}_{\text {diff }}$ in the diffusive zones. The function $\widetilde{\mathcal{G}}_{\text {diff }}\left(z, z^{\prime}, \varepsilon\right)$ must vanish for $\left|z-z^{\prime}\right| \gg \ell_{\mathrm{e}}^{Q}$ (see, e.g., Ref. 53). This imposes to cancel the "exponentially divergent" terms in Eq. (28), which requires ${ }^{37}$

$$
\begin{aligned}
& \left(\hat{\Sigma}_{3}+\check{G}_{L}\right)\left(\widetilde{g}_{L}-\hat{\Sigma}_{3}\right)=0, \\
& \left(\widetilde{g}_{L}+\hat{\Sigma}_{3}\right)\left(\hat{\Sigma}_{3}-\check{G}_{L}\right)=0, \\
& \left(\hat{\Sigma}_{3}-\check{G}_{R}\right)\left(\tilde{g}_{R}+\hat{\Sigma}_{3}\right)=0, \\
& \left(\widetilde{g}_{R}-\hat{\Sigma}_{3}\right)\left(\hat{\Sigma}_{3}+\check{G}_{R}\right)=0 .
\end{aligned}
$$

For $z \rightarrow \mp d_{L(R)}$ we obtain from Eqs. (28)-(33) that $\tilde{\mathcal{G}}$ finally approaches

$$
\begin{aligned}
\tilde{\mathcal{G}}_{\text {diff }}\left(z, z^{\prime}, \varepsilon\right)= & -i \pi \exp \left(-\frac{\left|z-z^{\prime}\right|}{2 \overline{\bar{v}}_{L(R)} \tau_{Q}}\right) \\
& \times\left(\check{G}_{L(R)}+\operatorname{sgn}\left(z-z^{\prime}\right) \hat{\Sigma}_{3}\right)
\end{aligned}
$$

so that $\widetilde{g}_{L(R)}\left(z, z^{\prime}=z, \varepsilon\right)$ tends to $\check{G}_{L(R)}$. As required, the expression (34) of $\widetilde{\mathcal{G}}_{\text {diff }}$ does not depend on the exact choice of the coordinate $d_{L(R)}$ and vanishes for $\left|z-z^{\prime}\right| \gg \ell_{\mathrm{e}}^{Q}$. Equations (28)-(33) indicate that the decay length for the isotropization of $\tilde{g}_{L(R)}\left(z, z^{\prime}=z, \varepsilon\right)$ is $\max _{n}\left[\left(2 m\left[E_{F, L(R)}-E_{n}\right]\right)^{1 / 2} \tau_{L(R)}\right]=\ell_{\mathrm{e}}^{L(R)}$, as anticipated above. Moreover, inserting Eq. (34) into Eq. (11) leads to an expression of $G$ whose semiclassical and isotropic average corresponds to $\check{G}_{L(R)}$, as expected. ${ }^{54}$ Importantly, from Eqs. (28)-(33), one sees explicitly that $\widetilde{\mathcal{G}}$ is smooth on a scale of the Fermi wavelength, which justifies $a$ posteriori the use of the approximated Eqs. (13) and (14) in this section.

\section{MATRIX CURRENT AND GENERAL BOUNDARY CONDITIONS}

Our purpose is to establish a relation between $\check{G}_{L}$ and $\check{G}_{R}$. To complete this task, it is convenient to introduce the matrix current $^{37}$ 


$$
\check{I}(z, \varepsilon)=\left.\frac{e^{2} \hbar}{\pi m} \int d \rho\left(\frac{\partial}{\partial z}-\frac{\partial}{\partial z^{\prime}}\right) G\left(\vec{r}, \vec{r}^{\prime}, \varepsilon\right)\right|_{\vec{r}=\vec{r}^{\prime}} .
$$

This quantity characterizes the transport properties of the circuit for coordinate $z$ and energy $\varepsilon$. It contains information on the charge current (see Sec. VII E) but also on the flows of spins and electron-hole coherence. Note that in this paper, $e$ denotes the absolute value of the electron charge. Using Eq. (11) and the orthonormalization of the transverse wave functions $\chi_{n}^{\sigma}$, the matrix current is written as

$$
\check{I}(z, \varepsilon)=2 i G_{q} \operatorname{Tr}_{n, s}\left[\hat{\Sigma}_{3} \widetilde{\mathcal{G}}(z, z, \varepsilon)\right] / \pi .
$$

for $z<-b_{L}$ or $z>b_{R}$. Here $\operatorname{Tr}_{n, s}$ denotes the trace in the $\underline{\mathcal{E}}$ subspace and $G_{q} \equiv e^{2} / 2 \pi \hbar$ is the conductance quantum. Inside the isotropization zones, using Eq. (28), one obtains ${ }^{49}$

$$
\check{I}(z, \varepsilon)=2 G_{q} \operatorname{Tr}_{n, s}\left[\hat{\Sigma}_{3} \widetilde{P}_{Q}\left[\lambda_{Q}(z)\right] \widetilde{g}_{Q} \widetilde{P}_{Q}\left[-\lambda_{Q}(z)\right]\right] .
$$

Considering that $\widetilde{P}_{Q}(z)$ has a structure in the $\mathcal{E}$ subspace only and that $\widetilde{P}_{Q}\left[-\lambda_{Q}(z)\right] \hat{\Sigma}_{3} \widetilde{P}_{Q}\left[\lambda_{Q}(z)\right]=\hat{\Sigma}_{3}$, one finds

$$
\check{I}(z, \varepsilon)=2 G_{q} \operatorname{Tr}_{n, s}\left[\hat{\Sigma}_{3} \widetilde{g}_{L(R)}\right]=\check{I}_{L(R)}(\varepsilon)
$$

at any point in the left (right) isotropization zone. We conclude that, quite generally, the matrix current is conserved inside each isotropization zone. We will see in next paragraph that this property is crucial to derive the BCIGF.

In order to express $\widetilde{g}_{L}$ in terms of $\check{G}_{L}$ and $\check{G}_{R}$, and $\bar{M}$, we multiply Eq. (30) by $\check{G}_{L}$ from the left and Eq. (32) by $\check{G}_{L} \bar{M}^{\dagger}$ from the left and by $\left(\bar{M}^{\dagger}\right)^{-1}$ from the right. Then, we add up the two resulting equations after simplifications based on Eqs. (22), (24), and (27). This leads to

$$
\check{I}_{L}(\varepsilon)=2 G_{q} \operatorname{Tr}_{n, s}\left[2 \widetilde{D}_{L}^{-1}\left(\check{G}_{L} \hat{\Sigma}_{3}+\mathbf{1}\right)-1\right]
$$

with $\widetilde{D}_{L}=\mathbf{1}+\check{G}_{L} \bar{M}^{\dagger} \check{G}_{R} \bar{M}$. A similar calculation leads to

$$
\check{I}_{R}(\varepsilon)=2 G_{q} \operatorname{Tr}_{n, s}\left[2 \widetilde{D}_{R}^{-1}\left(\check{G}_{R} \hat{\Sigma}_{3}-\mathbf{1}\right)+1\right]
$$

with $\widetilde{D}_{R}=\mathbf{1}+\check{G}_{R}\left(\bar{M}^{\dagger}\right)^{-1} \check{G}_{L} \bar{M}^{-1}$. Equations (39) and (40) represent the most general expression for $\check{I}_{L(R)}(\varepsilon)$ in terms of the isotropic Green's functions $\check{G}_{L(R)}$ and the transfer matrix $\bar{M}$. The conservation of the matrix current up to the beginning $z=\mp d_{L(R)}$ of the diffusive zones allows to identify these expressions with

$$
\check{I}_{L(R)}(\varepsilon)=-\left.\frac{A}{\rho_{L[R]}} \check{G}(z, \varepsilon) \frac{\partial \check{G}(z, \varepsilon)}{\partial z}\right|_{z=\mp d_{L(R)}} .
$$

Here, $\rho_{L(R)}$ denotes the resistivity of conductor $L(R)$ and $A$ the junction area. Formally speaking, Eqs. (39)-(41) complete our task of finding the general BCIGF for spindependent and diffusive metallic interfaces. We recall that to derive these equations, we have assumed a weak exchange field in ferromagnets $\left(E_{e x} \ll E_{F}\right)$, as required to reach the diffusive limit [see Eq. (10)]. However, we have made no restriction on the structure of the contact transfer matrix $\bar{M}$. In particular, $\bar{M}$ can be arbitrarily spin polarized and it is not necessarily spin conserving or channel conserving. However, at this stage, a concrete calculation requires the knowledge of the full $\bar{M}$ (or equivalently the full scattering matrix). Usually this information is not available for realistic interfaces and one has to reduce Eqs. (39) and (40) to simple expressions, using some simplifying assumptions. For a spinindependent tunnel interface, Eqs. (39) and (40) can be expressed in terms of the contact tunnel conductance $G_{T}$ only, which is a formidable simplification. ${ }^{17}$ Another possibility is to disregard superconducting correlations. In this case, Eqs. (39) and (40) lead to the normal-state BCIGF introduced in Refs. 35 and 36 (see Appendix $\mathrm{C}$ for details). The normalstate BCIGF involve the conductance $G_{T}$ but also a coefficient $G_{M R}$ which accounts for the spin dependence of the contact scattering probabilities, and the transmission and reflection mixing conductances $G_{m i x}^{t}$ and $G_{m i x}^{L(R), r}$ which account for spin-torque effects and interfacial effective fields. ${ }^{55} \mathrm{We}$ will show below that for a circuit enclosing superconducting elements, the BCIGF can also be simplified in various limits.

Note that since the transition between the ballistic, isotropization, and diffusive zones is smooth, the choice of the coordinates $d_{L(R)}$ and $c_{L(R)}$ in Fig. 1 is somewhat arbitrary, i.e., defined only up to an uncertainty of the order of $\ell_{\mathrm{e}}^{L(R)}$ or a fraction of $\ell_{\mathrm{e}}^{L(R)}$, respectively. However, one can check that this choice does not affect the BCIGF. First, a change in $c_{L}$ and $c_{R}$ by quantities $\delta c_{L}$ and $\delta c_{R}$ of the order of a fraction of $\ell_{\mathrm{e}}^{L(R)}$ requires to replace the matrix $\bar{M}$ appearing in Eqs. (39)-(41) by $\overline{\bar{A}}_{R} \bar{M} \overline{\bar{A}}_{L}$, where the matrices $\overline{\bar{A}}_{R}$ and $\overline{\bar{A}}_{L}$ have a nontrivial (i.e., diagonal) structure in the $\mathcal{E}$ subspace only, with diagonal elements $\overline{\bar{A}}_{L, n, s}=\exp \left[\begin{array}{llll}i & s & \delta c_{L} & k_{n}\end{array}\right]$ and $\overline{\bar{A}}_{R, n, s}$ $=\exp \left[\begin{array}{llll}i & s & \delta c_{R} & k_{n}\end{array}\right]$. Since $\bar{G}_{L(R)}$ commutes with $\overline{\bar{A}}_{R[L]}$, this leaves the BCIGF unchanged. Second, due to Eqs. (30)-(33), the BCIGF do not depend either on the exact values of $d_{L}$ and $d_{R}$.

\section{CASE OF A WEAKLY SPIN-DEPENDENT $S / F$ CONTACT}

\section{A. Perturbation scheme}

In the next sections, we assume that the transverse channel index $n$ and the spin index $\sigma=\uparrow, \downarrow$ corresponding to spin components along $\vec{Z}$ are conserved when electrons are scattered by the potential barrier $\bar{V}_{b}$ between the two ballistic zones [we use for instance $\bar{V}_{b}(z, \vec{\rho})=V_{0}(z) \check{\sigma}_{0}+V_{1}(z) \check{\sigma}_{Z}$ ]. In this case, one can describe the scattering properties of the barrier with parameters $T_{n}, P_{n}, \varphi_{n}^{L(R)}$, and $d \varphi_{n}^{L(R)}$ defined from

$$
\left|t_{L(R), n \sigma}\right|^{2}=T_{n}\left(1+\sigma P_{n}\right)
$$

and

$$
\arg \left(r_{L(R), n \sigma}\right)=\varphi_{n}^{L(R)}+\sigma\left(d \varphi_{n}^{L(R)} / 2\right)
$$

with $t_{L(R), n \sigma}$ the transmission amplitude from side $L(R)$ to side $R(L)$ of the barrier and $r_{L(R), n \sigma}$ the reflection amplitude at side $L(R)$. The parameter $P_{n}$ corresponds to the spin polarization of the transmission probability $\left|t_{R(L), n \sigma}\right|^{2}$. The parameters $d \varphi_{n}^{L}$ and $d \varphi_{n}^{R}$ characterize the spin dependence of 
interfacial phase shifts (SDIPS), also called in other references spin-mixing angle. ${ }^{19-21}$ In our model, $P_{n}$ and $d \varphi_{n}^{L(R)}$ can be finite due to the spin-dependent interface potential $\bar{V}_{b}$. Due to flux conservation and spin conservation along $\vec{Z}$, the parameters $T_{n}, P_{n}, \varphi_{n}^{L(R)}$, and $d \varphi_{n}^{L(R)}$ are sufficient to determine the value of the whole $\mathcal{M}^{e}$ matrix (see Appendix A for details). Then, using Eq. (23), one can obtain an expression for $\bar{M}$. We will work below at first order in $P_{n}$ and $d \varphi_{n}^{L(R)}$. In this case, $\bar{M}$ can be decomposed as

$$
\bar{M}=\hat{M}^{0}(\mathbf{1}+\delta \bar{X}) .
$$

The $n$th diagonal element of $\hat{M}^{0}$ in the transverse channel subspace has the form, in the propagation direction subspace

$$
\hat{M}_{n, n}^{0}=\left[\begin{array}{cc}
\frac{i e^{i\left(\varphi_{n}^{L}+\varphi_{n}^{R}\right) / 2}}{\sqrt{T_{n}}} & -i e^{i\left(\varphi_{n}^{R}-\varphi_{n}^{L}\right) / 2} \sqrt{\frac{R_{n}}{T_{n}}} \\
i e^{i\left(\varphi_{n}^{L}-\varphi_{n}^{R}\right) / 2} \sqrt{\frac{R_{n}}{T_{n}}} & -\frac{i e^{-i\left(\varphi_{n}^{L}+\varphi_{n}^{R}\right) / 2}}{\sqrt{T_{n}}}
\end{array}\right] \check{\sigma}_{0}
$$

with $R_{n}=1-T_{n}$. Accordingly, the matrix $\delta \bar{X}$ is, in the propagation direction subspace

$$
\delta \bar{X}=\left[\begin{array}{cc}
\delta \bar{X}_{++} & \delta \bar{X}_{+-} \\
\delta \bar{X}_{+-}^{*} & -\delta \bar{X}_{++}
\end{array}\right]
$$

with

$$
\delta \bar{X}_{n+, n+}=\frac{i \check{\sigma}_{Z}}{4 T_{n}}\left[T_{n} d \varphi_{n}^{L}+\left(2-T_{n}\right) d \varphi_{n}^{R}\right]
$$

and

$$
\delta \bar{X}_{n+, n-}=\frac{\check{\sigma}_{Z} e^{-i \varphi_{n}^{L}}}{2}\left(\frac{P_{n}}{\sqrt{R_{n}}}-i \frac{\sqrt{R_{n}}}{T_{n}} d \varphi_{n}^{R}\right) .
$$

One can check that Eqs. (44)-(48) are consistent with Eq. (24). Due to Eq. (23), the matrices $\hat{M}^{0}$ and $\delta \bar{X}$ are proportional to the identity in the Nambu subspace. The matrix $\hat{M}^{0}$ is determined by the parameters $T_{n}$ and $\varphi_{n}^{L(R)}$. It has a structure in the $\mathcal{E}$ subspace only. In contrast, $\delta \bar{X}$ is a first-order term in $P_{n}$ and $d \varphi_{n}^{L(R)}$ with a structure in the $\mathcal{E}$ subspace but also in the spin subspace. We conclude that the matrices $\hat{M}^{0}$ and $\check{G}_{L(R)}$ commute with each other whereas $\delta \bar{X}$ commutes neither with $\hat{M}^{0}$ nor with $\check{G}_{L(R)}$.

We want to express the matrix current of the isotropization zones as

$$
\check{I}_{L(R)}(\varepsilon)=\check{I}_{L(R)}^{0}(\varepsilon)+\check{I}_{L(R)}^{1}(\varepsilon)
$$

with $\check{I}_{L(R)}^{0}(\varepsilon)$ and $\check{I}_{L(R)}^{(1)}(\varepsilon)$ as zeroth and first-order terms in $\delta \bar{X}$, respectively. We will mainly focus on the calculation of $\check{I}_{L}(\varepsilon)$ because the calculation of $\check{I}_{R}(\varepsilon)$ is similar. To develop the expression (39) of $\check{I}_{L}(\varepsilon)$, one can use

$$
\widetilde{D}_{L}^{-1}=\widetilde{J}-\widetilde{J} \delta \tilde{V} \widetilde{J}+o\left(\delta \widetilde{V}^{2}\right)
$$

with

$$
\begin{gathered}
\delta \widetilde{V}=\check{G}_{L} \check{G}_{R} \hat{Q}_{0} \delta \bar{X}+\check{G}_{L} \delta \bar{X}^{\dagger} \hat{Q}_{0} \check{G}_{R}, \\
\hat{Q}_{0}=\left(\hat{M}^{0}\right)^{\dagger} \hat{M}^{0}
\end{gathered}
$$

and

$$
\widetilde{J}=\left(\mathbf{1}+\hat{Q}_{0} \check{G}_{L} \check{G}_{R}\right)^{-1} .
$$

For later use, we note that

$$
\widetilde{J}=\frac{\check{G}_{R} \check{G}_{L}+\hat{Q}_{0}^{-1}}{\left\{\check{G}_{L}, \check{G}_{R}\right\}+\hat{Q}_{0}+\hat{Q}_{0}^{-1}} .
$$

In the next sections, we will substitute Eq. (50) into Eq. (39), to express $\check{I}_{L}^{(0)}(\varepsilon)$ and $\check{I}_{L}^{(1)}(\varepsilon)$ in terms of the scattering parameters of the contact.

\section{B. Zeroth order component of the matrix current}

We first discuss the conservation of the zeroth-order matrix current across the contact. From Eqs. (22) and (38), one finds $\check{I}_{R}^{0}(\varepsilon)=2 i G_{q} \operatorname{Tr}_{n, s}\left[\hat{\Sigma}_{3} \hat{M}^{0} \widetilde{g}_{L}\left(\hat{M}^{0}\right)^{\dagger}\right] / \pi$. Since $\hat{M}^{0}$ has a structure in the $\underline{\mathcal{E}}$ subspace only, the cyclic property of the trace $\operatorname{Tr}_{n, s}$ yields $\check{I}_{L}^{0}(\varepsilon)=\check{I}_{R}^{0}(\varepsilon)=\check{I}^{0}(\varepsilon)$. Hence, the matrix current is conserved across the contact in the spin-degenerate case.

We now calculate $\check{I}^{(0)}(\varepsilon)$. Since $\hat{M}^{0}$ commutes with $\check{G}_{L(R)}$, Eq. (39) gives

$$
\check{I}^{(0)}(\varepsilon)=2 G_{q} \operatorname{Tr}_{n, s}\left[\widetilde{J}\left(2 \hat{\Sigma}_{3} \check{G}_{L}+\mathbf{1}-\hat{Q}_{0} \check{G}_{L} \check{G}_{R}\right)\right] .
$$

From Eq. (45), one finds

$$
\hat{Q}_{0}=-2 \hat{T}_{0}^{-1} \sqrt{\mathbf{1}-\hat{T}_{0}}\left[\cos \left(\varphi_{n}^{L}\right) \hat{\Sigma}_{1}+\sin \left(\varphi_{n}^{L}\right) \hat{\Sigma}_{2}\right]+\left(2 \hat{T}_{0}^{-1}-1\right) \hat{\Sigma}_{0}
$$

and

$$
\hat{Q}_{0}^{-1}=\hat{\Sigma}_{3} \hat{Q}_{0} \hat{\Sigma}_{3}
$$

In Eq. (56), the matrices $\hat{\Sigma}_{0}, \hat{\Sigma}_{1}$, and $\hat{\Sigma}_{2}$ refer to the identity, the first and second Pauli matrices in the propagation direction subspace, respectively. We use $\left(\hat{T}_{0}\right)_{n s, m s^{\prime}}^{\nu \sigma, \nu^{\prime} \sigma^{\prime}}$ $=T_{n} \delta_{s s^{\prime}} \delta_{n m} \delta_{\sigma \sigma^{\prime}} \delta_{\nu \nu^{\prime}} \mathbf{1}_{K}$. We find that $\hat{Q}_{0}+\hat{Q}_{0}^{-1}$ has a diagonal structure in the propagation direction space. Therefore, using expression (54) for $\widetilde{J}$, and performing the trace over the channel and propagation direction indices, we obtain

$$
\check{I}^{(0)}(\varepsilon)=4 G_{q} \sum_{n} \frac{T_{n}\left[\check{G}_{R}, \check{G}_{L}\right]}{4+T_{n}\left(\left\{\check{G}_{L}, \check{G}_{R}\right\}-2\right)} .
$$

Equation (58) corresponds to the expression obtained in Ref. 37 for a spin-independent contact. ${ }^{56}$ This expression does not involve any scattering phase shift.

\section{First-order component of the matrix current}

We now concentrate on the contribution $\check{I}_{L(R)}^{(1)}(\varepsilon)$ to the matrix current to first order in $\delta \bar{X}$. Equations (39) and (50) lead to 


$$
\check{I}_{L}^{(1)}(\varepsilon)=-4 G_{q} \operatorname{Tr}_{n, s}\left[\widetilde{J} \delta \tilde{V} \widetilde{J}\left(\mathbf{1}+\hat{\Sigma}_{3} \check{G}_{L}\right)\right]
$$

with $\delta \tilde{V}$ given by Eq. (51). Using Eqs. (46)-(48), (56), and (57), and performing the trace over the transverse channel and propagation direction indices (see Appendix B for details), one finds

$$
\begin{aligned}
\check{I}_{L}^{(1)}(\varepsilon)= & 2 G_{q} \sum_{n}\left(4+T_{n}\left(\left\{\check{G}_{L}, \check{G}_{R}\right\}-2\right)\right)^{-1} \\
& \times\left(4 T_{n} P_{n}\left[\left\{\check{\sigma}_{Z}, \check{G}_{R}\right\}, \check{G}_{L}\right]-i 8 R_{n} d \varphi_{n}^{L}\left[\check{\sigma}_{Z}, \check{G}_{L}\right]\right. \\
& +i T_{n}\left(T_{n} d \varphi_{n}^{L}+\left(2-T_{n}\right) d \varphi_{n}^{R}\right)\left[\check{G}_{R}\left[\check{\sigma}_{Z}, \check{G}_{R}\right], \check{G}_{L}\right] \\
& \left.-i T_{n}\left(T_{n} d \varphi_{n}^{R}+\left(2-T_{n}\right) d \varphi_{n}^{L}\right)\left[\left[\check{\sigma}_{Z}, \check{G}_{R}\right] \check{G}_{L}, \check{G}_{L}\right]\right) \\
& \times\left(4+T_{n}\left(\left\{\check{G}_{L}, \check{G}_{R}\right\}-2\right)\right)^{-1} .
\end{aligned}
$$

A comparison between Eqs. (39) and (40) indicates that the expression of $\check{I}_{R}^{(1)}(\varepsilon)$ can be obtained by multiplying the expression (60) of $\check{I}_{L}^{(1)}(\varepsilon)$ by -1 , replacing $d \varphi_{n}^{L(R)}$ by $d \varphi_{n}^{R(L)}$, and $\check{G}_{L(R)}$ by $\check{G}_{R(L)}$. Note that the expressions of $\check{I}_{L}^{(1)}(\varepsilon)$ and $\check{I}_{R}^{(1)}(\varepsilon)$ involve the SDIPS parameters $d \varphi_{n}^{L}$ and $d \varphi_{n}^{R}$ but not the spinaveraged phases $\varphi_{n}^{L}$ and $\varphi_{n}^{R}$.

\section{Expression of the matrix current in the tunnel limit}

We now assume that the contact is a tunnel barrier $\left(T_{n}\right.$ $\ll 1$ ), which seems reasonable considering the band-structure mismatch between most $S$ and $F$ materials. At first order in $T_{n}$, the matrix currents $\check{I}_{L(R)}(\varepsilon)$ take the very transparent form

$$
\begin{aligned}
2 \check{I}_{L}(\varepsilon)= & G_{T}\left[\check{G}_{R}, \check{G}_{L}\right]+G_{M R}\left[\left\{\check{\sigma}_{Z}, \check{G}_{R}\right\}, \check{G}_{L}\right]+i G_{\phi}^{L}\left[\check{\sigma}_{Z}, \check{G}_{L}\right] \\
& -i G_{\chi}^{L}\left[\left[\check{\sigma}_{Z}, \check{G}_{R}\right] \check{G}_{L}, \check{G}_{L}\right]+i G_{\chi}^{R}\left[\check{G}_{R}\left[\check{\sigma}_{Z}, \check{G}_{R}\right], \check{G}_{L}\right]
\end{aligned}
$$

and

$$
\begin{aligned}
2 \check{I}_{R}(\varepsilon)= & G_{T}\left[\check{G}_{R}, \check{G}_{L}\right]+G_{M R}\left[\check{G}_{R},\left\{\check{\sigma}_{Z}, \check{G}_{L}\right\}\right]-i G_{\phi}^{R}\left[\check{\sigma}_{Z}, \check{G}_{R}\right] \\
& +i G_{\chi}^{R}\left[\left[\check{\sigma}_{Z}, \check{G}_{L}\right] \check{G}_{R}, \check{G}_{R}\right]-i G_{\chi}^{L}\left[\check{G}_{L}\left[\check{\sigma}_{Z}, \check{G}_{L}\right], \check{G}_{R}\right] .
\end{aligned}
$$

We have introduced above the conductance parameters ${ }^{58}$

$$
\begin{gathered}
G_{T} / G_{q}=2 \sum_{n} T_{n}, \\
G_{M R} / G_{q}=\sum_{n} T_{n} P_{n}, \\
G_{\phi}^{L(R)} / G_{q}=2 \sum_{n}\left(T_{n}-1\right) d \varphi_{n}^{L(R)}, \\
G_{\chi}^{L(R)} / G_{q}=\sum_{n} T_{n} d \varphi_{n}^{L(R)} / 2 .
\end{gathered}
$$

The values of the coefficients $G_{T}, G_{M R}, G_{\phi}^{L(R)}$, and $G_{\chi}^{L(R)}$ are difficult to predict because they depend on the detailed microscopic structure of the interface. These parameters can, in principle, be large compared to $G_{q}$ because, although the derivation of Eqs. (61) and (62) assumes that $T_{n}, P_{n}$, and $d \varphi_{n}^{L(R)}$ are small, the definitions, Eqs. (63)-(66), involve a summation on a numerous number of channels. The parameter $G_{M R}$ can be finite when $P_{n} \neq 0$ and the parameters $G_{\phi}^{L(R)}$ and $G_{\chi}^{L(R)}$ can be finite due to the SDIPS. From Eqs. (63)-(66), $G_{\chi}^{L}$ and $G_{\chi}^{R}$ are likely to be small compared to $G_{T}$ and $G_{\phi}^{L(R)}$. This is why these coefficients were disregarded so far for studying the effects of the SDIPS on the superconducting proximity effect. In contrast, it is possible to have $G_{\phi}^{L(R)}>G_{T}$ as well as $G_{\phi}^{L(R)}<G_{T}$, using a spin-dependent interface potential $\bar{V}_{b}{ }^{24}$ We also note that the hypothesis $P_{n}$ $\ll 1$ imposes $G_{M R} \ll G_{T}$. We have checked that in the normalstate limit, Eqs. (61) and (62) agree with the boundary conditions introduced in Refs. 35 and 36 provided the reflectionand transmission-mixing conductances $G_{m i x}^{L(R), r}$ and $G_{m i x}^{t}$ appearing in these boundary conditions are replaced by their developments at first order in $T_{n}, P_{n}$, and $d \varphi_{n}^{L(R)}$, i.e.,

$$
G_{\text {mix }}^{L(R), r} \rightarrow\left(G_{T}+i G_{\phi}^{L(R)}\right) / 2
$$

and

$$
G_{m i x}^{t} \rightarrow\left(G_{T} / 2\right)+i\left(G_{\chi}^{L}+G_{\chi}^{R}\right)
$$

(see Appendix C for details).

We now briefly review the physical effects of the coefficients $G_{T}, G_{M R}$, and $G_{\phi}^{L(R)}$. The term in $G_{T}$ in Eqs. (61) and (62) corresponds to the term derived in Ref. 17 for superconducting/normal-metal interfaces. This term is responsible for the superconducting proximity effect occurring in a normal-metal layer or a ferromagnetic layer in contact with a superconductor. The parameter $G_{M R}$ accounts for the spin dependence of the contact tunnel probabilities and thus leads to magnetoresistance effects. ${ }^{28,29,32}$ In a ferromagnet $F$ subject to the proximity effect, the ferromagnetic exchange field causes spatial oscillations of the isotropic Green's function $\check{G}$, which results, e.g., in spatial oscillations of the density of states of $F$. It has been shown that the $G_{\phi}^{L(R)}$ terms can shift these oscillations. ${ }^{24-26}$ The $G_{\phi}^{L(R)}$ terms also induce Zeeman effective fields inside thin superconducting or normalmetal layers. ${ }^{22-24}$ In principle, in noncollinear geometries enclosing several ferromagnetic elements with noncollinear magnetizations, the SDIPS terms can induce spin-precession effects.

Note that, so far, we have considered that the interface potential $\bar{V}_{b}$ is spin polarized along the $\vec{Z}$ direction. In the general case, due to interface effects, it is possible that the spin-dependent part of the interface potential $\bar{V}_{b}$ is polarized along a direction $\vec{m}$ different from the bulk exchange-field direction of contacts $L$ or $R$. It is also possible that the contact belongs to a circuit enclosing several ferromagnets with noncollinear magnetizations, or ferromagnets with a spatially dependent magnetization direction. In these cases, Eqs. (39)-(41) are still valid. One can furthermore generalize the BCIGF Eqs. (60)-(62) to an arbitrary spin reference frame $\left(\check{\sigma}_{X}, \check{\sigma}_{Y}, \check{\sigma}_{Z}\right)$ by replacing $\check{\sigma}_{Z}$ by $\vec{m} \cdot \check{\vec{\sigma}}$.

As we have already explained in Sec. IV, the use of transfer matrices for the derivation of Eqs. (61)-(66) allows to obtain results for the $T_{n} \rightarrow 0$ limit, which must be performed after an explicit calculation of the BCIGF. From Eq. (65), 
even if a channel $n$ is perfectly reflected at the $L / R$ boundary, it can contribute to the matrix current due to the spin dependence of the reflection phase $d \varphi_{n}^{L(R)}$. We will recover this result in Sec. VIII A for a S/FI contact, using an approach without transfer matrices.

\section{E. Discussion on the matrix current conservation and the spin-dependent circuit theory}

In this section, we discuss the nonconservation of the matrix current in the general case. We have already seen in Sec. VII $B$ that the full matrix current is conserved across an interface in the spin-degenerate case. In the spin-dependent situation, one finds from Eqs. (22) and (38) that $\check{I}_{R}(\varepsilon)$ $=2 i G_{q} \operatorname{Tr}_{n, s}\left[\hat{\Sigma}_{3} \bar{M} \widetilde{g}_{L}(\bar{M})^{\dagger}\right] / \pi$. Since $\bar{M}$ has a structure in the spin subspace, the cyclic property of the trace $\operatorname{Tr}_{n, s}$ cannot be used anymore to relate $\check{I}_{L}(\varepsilon)$ and $\check{I}_{R}(\varepsilon)$. Hence, nothing imposes $\check{I}_{L}(\varepsilon)=\check{I}_{R}(\varepsilon)$ in the general case. Reference 24 illustrates that in the case of a simple $S / F$ bilayer with a homogeneous magnetization in $F, \check{I}_{L}(\varepsilon) \neq \check{I}_{R}(\varepsilon)$ is already possible. Note that $\check{I}_{L}(\varepsilon) \neq \check{I}_{R}(\varepsilon)$ does not violate particle current conservation through the interface although the average current flowing at side $Q$ of the contact is determined by $\check{I}_{Q}$, i.e.,

$$
\left\langle I_{Q}\right\rangle=\frac{1}{16 \mathrm{e}} \int_{-\infty}^{\infty} d \varepsilon \operatorname{Tr}_{\nu \sigma}\left\{\check{\tau}_{3} \check{I}_{Q}^{K}(\varepsilon)\right\} .
$$

Indeed, the above equation leads to

$$
\left\langle I_{L}\right\rangle=\frac{G_{q}}{8 \mathrm{e}} \int_{-\infty}^{\infty} d \varepsilon \operatorname{Tr}_{n s \nu \sigma}\left\{\check{\tau}_{3} \hat{\Sigma}_{3} \widetilde{g}_{L}^{K}\right\}
$$

and

$$
\left\langle I_{R}\right\rangle=\frac{G_{q}}{8 \mathrm{e}} \int_{-\infty}^{\infty} d \varepsilon \operatorname{Tr}_{n s \nu \sigma}\left\{\check{\tau}_{3} \hat{\Sigma}_{3} \bar{M} \widetilde{g}_{L}^{K} \bar{M}^{\dagger}\right\} .
$$

Since $\bar{M}$ is proportional to the identity in the Keldysh space, one can use the cyclic property of the trace $\operatorname{Tr}_{n s \nu \sigma}$ in the above equations, to show that $\left\langle I_{L}\right\rangle=\left\langle I_{R}\right\rangle$. It is important to point out that the nonconservation of the matrix current at the $L / R$ boundary does not affect the applicability of Eqs. (39)-(41). The fact that the matrix current is not conserved through a spin-dependent interface has the obvious reason that only charge conservation is required by fundamental laws whereas other quantities are not conserved in general. It depends on the symmetry of the Hamiltonian describing the barrier, which quantities are conserved in addition to charge. If the barrier potential is spin independent, all elements of the matrix current are conserved. In general, this is not the case anymore for spin-dependent barriers. An extreme case illustrating this situation is provided by an interface between a FI and a metal. In the FI, the concept of a matrix current does not even exist although the FI influences the adjacent metal due to the proximity effect. We will discuss this case in Sec. VIII

The BCIGF derived in this paper allow to generalize the "circuit theory" of Ref. 37 to the case of multiterminal cir- cuits which enclose superconductors, normal metals, ferromagnets, and ferromagnetic insulators. In the approach of circuit theory, a system is split up into reservoirs $r$ (voltage sources), connectors $c$ (contacts, interfaces), and nodes $n$ (small islands) in analogy to classical electric circuits. Each reservoir or node is characterized with an isotropic Green's function with no space dependence, which plays the role of a generalized potential. Circuit theory requires to apply generalized Kirchhoff's rules on the matrix current $\check{I}$. We have seen above that $\check{I}$ is not conserved through the contacts in the general case but this is not a problem since we know how to express the matrix current at both sides of the contact. We will note $\check{I}_{c}^{n}$ the matrix current flowing from the connector $c$ into node $n$, which is given by Eqs. (39) and (40). One must be careful to the fact that the matrix current is not conserved either inside the nodes due the terms on the right-hand side of the Usadel Eq. (E1). To compensate for the nonconservation of $\check{I}$ inside node $n$, one can introduce a leakage matrix current

$$
\check{I}_{\text {leakage }}^{n}=4 \pi G_{q} \nu_{0} V_{n}\left[-i \varepsilon \check{\tau}_{3}+\check{\Delta}+i E_{\text {ex }} \check{\sigma}_{Z}, \check{G}_{n}\right]
$$

which accounts for the "leakage" of quantities such as, for instance, electron-hole coherence or spin accumulation. In the above expression, $\check{G}_{n}, \check{\Delta}$, and $E_{e x}$, refer to the values of the isotropic Green's function, gap matrix, and exchange field inside $n$, and $V_{n}$ is the volume of the node. The leakage matrix current $\check{I}_{\text {leakage }}^{n}$ can be viewed as flowing from an effective "leakage terminal." It must occur in the generalized Kirchhoff's rule for node $n$, i.e.,

$$
\check{I}_{\text {leakage }}^{n}+\sum_{c} \check{I}_{c}^{n}=0
$$

with the index $c$ running on all the contacts connected to node $n$. We refer the reader to Refs. 37 and 59 for more details on circuit theory.

\section{CONTACT BETWEEN A METAL AND A FERROMAGNETIC INSULATOR}

\section{A. Boundary conditions}

In the case of a contact between a metal and a ferromagnetic insulator, one can perform a calculation similar to the one of the metallic case without using the transfer matrix $\bar{M}$ but a simpler "pseudotransfer" matrix $\overline{\mathrm{M}}$ which involves only reflection phases against the $F I$ (see definition below). This facilitates a development of the BCIGF at higher orders in the SDIPS parameters. We assume that the ferromagnetic insulator is located at the right side $(z>0)$ of the contact and that the left side $L$ is a BCS superconductor, a normal metal, or a ferromagnet, which satisfies Eq. (10). We divide $L$ into a ballistic zone, an isotropization zone and a diffusive zone like in Fig. 1. We directly consider the case where the contact locally conserves the transverse channel index and spins along $\vec{Z}$. In this case, one can introduce a reflection phase shift $\varphi_{n}+\sigma d \varphi_{n} / 2$ such that the left-going and right-going quasiparticle wave functions in the $n$th channel of $L$ are related by 


$$
\psi_{n,-}^{\nu, \sigma}\left(-c_{L}, \varepsilon\right)=e^{i\left(\varphi_{n}+\sigma d \varphi_{n} / 2\right)} \psi_{n, s+}^{\nu, \sigma}\left(-c_{L}, \varepsilon\right) .
$$

Using this relation, one can check that the calculations of Secs. IV-VI can be repeated by replacing the ballistic Green's function $\widetilde{g}_{R}$ by $\hat{\Sigma}_{1} \widetilde{g}_{L} \hat{\Sigma}_{1}, \check{G}_{R}$ by $\check{G}_{L}$, and the transfer matrix $\bar{M}$ by a pseudotransfer matrix $\overline{\mathrm{M}}=\hat{\mathbb{M}}^{0} \exp (\delta \overline{\mathrm{X}})$. The $n$th diagonal elements of $\hat{\mathrm{M}}^{0}$ and $\delta \overline{\mathbb{X}}$ in the transverse channel subspace write

$$
\hat{\mathbb{M}}_{n}^{0}=\left[\cos \left(\varphi_{n}\right) \hat{\Sigma}_{0}+i \sin \left(\varphi_{n}\right) \hat{\Sigma}_{3}\right] \check{\sigma}_{0}
$$

and

$$
\delta \overline{\mathrm{X}}_{n}=i d \varphi_{n} \hat{\Sigma}_{3} \check{\sigma}_{Z} / 2
$$

Since $\hat{\mathbb{M}}^{0}$ commutes with $\check{G}_{L}$ and $\delta \overline{\mathrm{X}}$ and $\left(\hat{\mathrm{M}}^{0}\right)^{\dagger} \hat{\mathrm{M}}^{0}=\mathbf{1}$, we find

$$
\check{I}_{L}(\varepsilon)=2 G_{q} \operatorname{Tr}_{n, s}\left[(\mathbf{1}+\delta \tilde{Y})^{-1}\left(\check{G}_{L} \hat{\Sigma}_{3}+\mathbf{1}\right)-\mathbf{1}\right]
$$

with

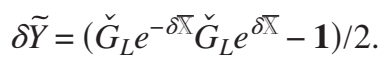

Hence, quite generally, the spin-averaged reflection phases $\varphi_{n}$ do not contribute to $\check{I}_{L}(\varepsilon)$. Equation (71) can be traced out numerically. Alternatively, one can achieve further analytical progress by expanding $\check{I}_{L}(\varepsilon)$ with respect to the spindependent part $\delta \widetilde{Y}$. We have $(\mathbf{1}+\delta \widetilde{Y})^{-1}=\mathbf{1}+\Sigma_{n}(-\delta \widetilde{Y})^{n}$. Therefore, at fourth order in $d \varphi_{n}$ we obtain

$$
\begin{aligned}
2 \check{I}_{L}(\varepsilon)= & i G_{\phi, 1}\left[\check{\sigma}_{Z}, \check{G}_{L}\right]+G_{\phi, 2}\left[\check{\sigma}_{Z}, \check{G}_{L} \check{\sigma}_{Z} \check{G}_{L}\right] \\
& +i G_{\phi, 3}\left[\check{\sigma}_{Z}, \check{G}_{L}\left(\check{\sigma}_{Z} \check{G}_{L}\right)^{2}\right]+G_{\phi, 4}\left[\check{\sigma}_{Z}, \check{G}_{L}\left(\check{\sigma}_{Z} \check{G}_{L}\right)^{3}\right]
\end{aligned}
$$

with the conductance parameters

$$
\begin{gathered}
G_{\phi, 1} / G_{q}=-2 \sum_{n} d \varphi_{n}-\sum_{n} d \varphi_{n}^{3} / 24, \\
G_{\phi, 2} / G_{q}=\sum_{n} d \varphi_{n}^{2} / 2+\sum_{n} d \varphi_{n}^{4} / 48 \\
G_{\phi, 3} / G_{q}=\sum_{n} d \varphi_{n}^{3} / 8 \\
G_{\phi, 4} / G_{q}=-\sum_{n} d \varphi_{n}^{4} / 32 .
\end{gathered}
$$

In the normal-state limit, we have checked that Eq. (73) agrees with the BCIGF presented in Refs. 35 and 36 (see Appendix $\mathrm{C}$ for a detailed comparison). The term in $G_{\phi, 1}$ of Eq. (73) has already been used in Refs. 22 and 23. At first order in $d \varphi_{n}$, it is the only term contributing to $\check{I}_{L}$, and it can be recovered from Eqs. (61) and (63)-(66) by using $T_{n}=0$ and $d \varphi_{n}^{L}=d \varphi_{n}$. At higher orders in $d \varphi_{n}$, the value of $G_{\phi, 1}$ is renormalized and new terms occur in the expression of $\check{I}_{L}$. The second-order term has a straightforward interpretation since it has exactly the same matrix structure as the selfenergy due to scattering by paramagnetic impurities in a normal metal ${ }^{60,61}$ or due to magnetic disorder along the $\vec{Z}$ direc- tion in a ferromagnet. ${ }^{62}$ The scattering of Cooper pairs at the spin-active interface leads to a coupling between spin-singlet and spin-triplet components, which, due to the random scattering at second order leads to pair breaking. In a similar fashion, we can understand the higher order terms in Eq. (73) as a result of multiple scattering at the $S / F I$ interface. Note that in this section, we have assumed that the FI side of the contact is magnetized along the $\vec{Z}$ direction. If the $F I$ is magnetized along a direction $\vec{m} \neq \vec{Z}$, one can describe the contact in the spin reference frame $\left(\check{\sigma}_{X}, \check{\sigma}_{Y}, \check{\sigma}_{Z}\right)$ by replacing $\check{\sigma}_{Z}$ by $\vec{m} \cdot \check{\breve{\sigma}}$ in Eq. (73)

\section{B. Example of a $S / F I$ bilayer}

To illustrate some effects of the $G_{\phi, i}$ coefficients, we now consider the case of a $S / F I$ bilayer, with $S$ located at $z$ $\in\left[0, d_{S}\right]$ and $F I$ at $z>d_{S}$. Throughout this section, we replace the energy $-i \varepsilon$ appearing in the Usadel equation by $-i \varepsilon+\Gamma$, where the phenomenological collision rate $\Gamma$ accounts for inelastic processes. ${ }^{63}$ Inside $S$, the retarded part of the isotropic Green's function can be parametrized with a so-called pairing angle $\Lambda_{\sigma}^{S}$ such that

$$
\check{G}^{r}=\left[\begin{array}{cccc}
\cos \left(\Lambda_{\uparrow}^{S}\right) & 0 & 0 & \sin \left(\Lambda_{\uparrow}^{S}\right) \\
0 & \cos \left(\Lambda_{\downarrow}^{S}\right) & \sin \left(\Lambda_{\downarrow}^{S}\right) & 0 \\
0 & \sin \left(\Lambda_{\downarrow}^{S}\right) & -\cos \left(\Lambda_{\downarrow}^{S}\right) & 0 \\
\sin \left(\Lambda_{\uparrow}^{S}\right) & 0 & 0 & -\cos \left(\Lambda_{\uparrow}^{S}\right)
\end{array}\right] .
$$

Let us first assume that $d_{S} \ll \xi_{S}$ so that one can use the quadratic approximation $\Lambda_{\sigma}^{S}(\varepsilon, x)=\Lambda_{\sigma}^{0}-\beta_{\sigma}\left(x / \xi_{S}\right)^{2}$ and a constant superconducting gap $\Delta(x)=\Delta_{0}$ inside $S$ (see, e.g., Ref. 24). For $z \in\left[0, d_{S}\right]$, the Usadel equations (see Appendix E) lead to

$$
\beta_{\sigma}=\frac{\Delta_{0} \cos \left(\Lambda_{\sigma}^{0}\right)+(i \varepsilon-\Gamma) \sin \left(\Lambda_{\sigma}^{0}\right)}{2 \Delta_{\mathrm{BCS}}} .
$$

We have introduced above the bulk BCS gap $\Delta_{\mathrm{BCS}}$ of $S$. The value of $\Lambda_{\sigma}^{0}$ can be found by identifying Eq. (78) with Eq. (73), i.e.,

$$
\begin{aligned}
2 \beta_{\sigma} d_{s} / \xi_{S}= & i \gamma_{\phi, 1} \sigma \sin \left(\Lambda_{\sigma}^{0}\right)+\gamma_{\phi, 2} \sin \left(2 \Lambda_{\sigma}^{0}\right)+i \gamma_{\phi, 3} \sigma \sin \left(3 \Lambda_{\sigma}^{0}\right) \\
& +\gamma_{\phi, 4} \sin \left(4 \Lambda_{\sigma}^{0}\right)
\end{aligned}
$$

(see Appendix D for details). We have introduced above $\gamma_{\phi, i}=G_{\phi, i} \xi_{S} \rho_{S} / A$. Note that the value of $\Delta_{0}$ must be calculated self-consistently with $\Lambda_{\sigma}^{0}$, see, e.g., Ref. 24. We will first consider the case $G_{\phi, 2}=G_{\phi, 3}=G_{\phi, 4}=0$, for which Eqs. (78) and (79) yield

$$
\Lambda_{\sigma}^{0}=\arctan \left(\frac{\Delta_{0}}{-i \varepsilon+\Gamma+i \gamma_{\phi, 1} \sigma \frac{\xi_{S}}{d_{s}} \Delta_{\mathrm{BCS}}}\right) .
$$

From the above equation, $G_{\phi, 1}$ induces an effective Zeeman field $H_{e f f}=2 i \gamma_{\phi, 1} \xi_{S} \Delta_{\mathrm{BCS}} / d_{s} g \mu_{B}$ inside a thin $S$ layer, like the $G_{\phi}^{L(R)}$ terms of Sec. VII D. ${ }^{22-24}$ The density of states (DOS) in the $S$ layer can be calculated as $N(\varepsilon, x)$ $=N_{0} \Sigma_{\sigma} \operatorname{Re}\left[\cos \left[\Lambda_{\sigma}^{S}(\varepsilon, x)\right]\right] / 2$, with $N_{0}$ the normal-state density 


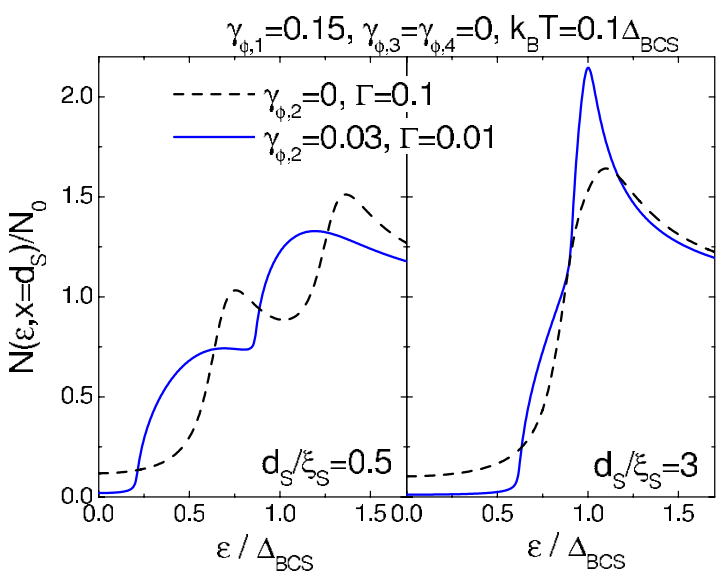

FIG. 2. (Color online) Density of states $N\left(\varepsilon, x=d_{S}\right)$ in a $S$ layer contacted to a $F I$. The black dashed lines correspond to $\Gamma=0.1$ and $\gamma_{\phi, 2}=0$, and the blue full lines correspond to $\Gamma=0.01$ and $\gamma_{\phi, 2}$ $=0.03$. The left panel corresponds to $d_{S} / \xi_{S}=0.5$ and the right panel corresponds to $d_{S} / \xi_{S}=3$. In all cases, we have used $\gamma_{\phi, 1}=0.15$, $\gamma_{\phi, 3}=\gamma_{\phi, 4}=0$, and $k_{B} T=0.1 \Delta_{\mathrm{BCS}}$.

of states. The $G_{\phi, 1}$-induced effective Zeeman field $H_{\text {eff }}$ splits the superconducting peaks of the DOS, as shown by the black dashed line in Fig. 2, left panel. Spin-splitting effects in $S / F$ systems were first intuited by De Gennes from a generalization of Cooper's argument. ${ }^{64,65}$ Later, Ref. 19 has confirmed from a quasiclassical approach that the SDIPS can induce a spin splitting of the DOS in a ballistic $S / F I$ bilayer with a thin $S$. However, the effect found by Tokuyasu et al. is qualitatively different from ours. Indeed, in the ballistic limit, Tokuyasu et al. find that the S/FI bilayer differs from a $S$ layer in an external field because the SDIPS induced spin-splitting effect depends upon the quasiparticle trajectory. In contrast, in the diffusive limit, we obtain a true effective Zeeman field $H_{\text {eff }}$ which appears directly in the spectral functions. On the experimental side, spin-splitted DOS were observed in superconducting $\mathrm{Al}$ layers contacted to different types of FI as soon as 1986 (see Refs. 66-69). However, the inadequacy of the ballistic approach of Tokuyasu et al. for modeling the actual experiments was pointed out in Ref. 69. In fact, most of the experiments on $\mathrm{Al} / \mathrm{FI}$ interfaces were interpreted by their authors in terms of a diffusive approach with no SDIPS and an internal Zeeman field added arbitrarily in the Al layer (see Refs. 68-70). Our approach provides a microscopic justification for the use of such an internal field. Remarkably, it was found experimentally ${ }^{69}$ that the internal field appearing in $S$ scales with $d_{s}^{-1}$, in agreement with our expression of $H_{\text {eff }}$.

We now discuss briefly the effects of the $G_{\phi, 2}, G_{\phi, 3}$, and $G_{\phi, 4}$ terms. Assuming $\Lambda_{\sigma}^{0} \ll 2 \pi$, the linearization of Eq. (79) leads to

$$
\Lambda_{\sigma}^{0}=\frac{\Delta_{0}}{-i \varepsilon+\Gamma+\xi_{S} \Delta_{\mathrm{BCS}} \frac{i\left(\gamma_{\phi, 1}+3 \gamma_{\phi, 3}\right) \sigma+2 \gamma_{\phi, 2}+4 \gamma_{\phi, 4}}{d_{s}}} .
$$

Therefore, in this limit, $G_{\phi, 3}$ contributes to the Zeeman effective field like $G_{\phi, 1}$. Moreover, the coefficients $G_{\phi, 2}$ and

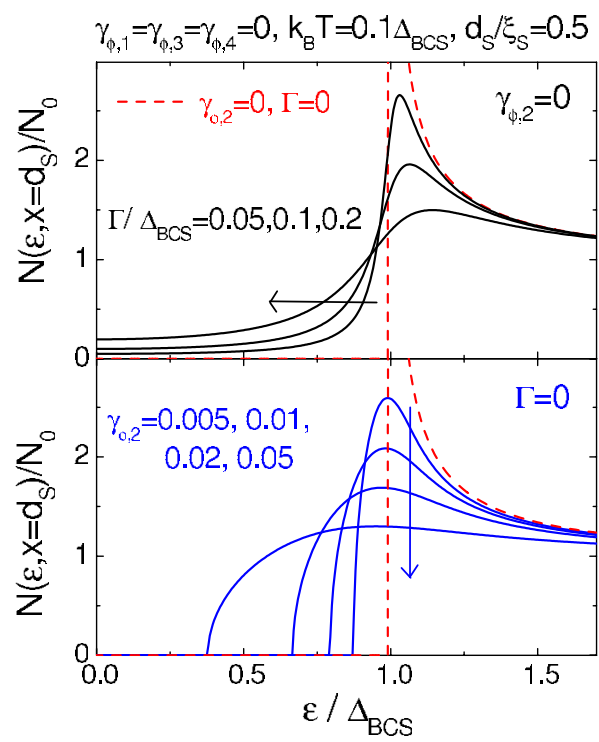

FIG. 3. (Color online) Top panel: density of states $N\left(\varepsilon, x=d_{S}\right)$ in a $S$ layer contacted to a $F I$. The red dashed line corresponds to $\Gamma$ $=0$ and $\gamma_{\phi, 2}=0$ in both panels. The black full lines in the top panel correspond to $\Gamma \neq 0$ and $\gamma_{\phi, 2}=0$, and the blue full lines in the bottom panel correspond to $\Gamma=0$ and $\gamma_{\phi, 2} \neq 0$. In all cases, we have used $d_{S} / \xi_{S}=0.5, \gamma_{\phi, 1}=\gamma_{\phi, 3}=\gamma_{\phi, 4}=0$, and $k_{B} T=0.1 \Delta_{\mathrm{BCS}}$.

$G_{\phi, 4}$ lead to a decoherence effect similar to the decoherence induced by the $\Gamma$ term. However, it is clear from Eq. (79) that this picture is not valid in the general case. Let us focus on the effect of $G_{\phi, 2}$. From Eq. (79), in the nonlinearized limit, $\gamma_{\phi, 2}$ occurs together with a $\sin \left(2 \Lambda_{\sigma}^{0}\right)$ in the expression of $\beta_{\sigma}$. Therefore, as already pointed out in Sec. VIII A, in the general case, it is more relevant to compare the effect of $\gamma_{\phi, 2}$ to that of paramagnetic impurities which would be diluted inside $S$. The analogy to magnetic disorder can be understood as arising due to successive reflections on the $S / F I$ interface with random spin-dependent phase shifts. To study the effect of $\gamma_{\phi, 2}$ in the general case, we have calculated the density of states $N(\varepsilon, x)$ numerically. Our code takes into account the self-consistency of the superconducting gap $\Delta(x)$ in the $S$ layer and is valid for arbitrary values of $d_{S} \cdot{ }^{71}$ Figure 3 compares the effect of $\Gamma \neq 0$ (top panel) with the effect of $G_{\phi, 2} \neq 0$ (bottom panel), for $G_{\phi, 1}=0$. As expected, we find that the effect of $G_{\phi, 2}$ on the DOS of a thin $S$ is quite similar to the effect of paramagnetic impurities which would be diluted inside the bulk of $S .^{72,73}$ First, a weak $G_{\phi, 2}$ widens the BCS peak in a way which is qualitatively different from $\Gamma$ since the curvature of the DOS for $\varepsilon<\Delta_{\mathrm{BCS}}$ has opposite signs in the two cases. Second, even a very small $\Gamma$ leads to a finite zero-energy DOS whereas a small $G_{\phi, 2}$ reduces the gap appearing in the DOS but preserves $N(\varepsilon, x)=0$ for small energies. For larger values of $G_{\phi, 2}$, we expect a gap suppression in the DOS (not shown in Fig. 3). Note that in Fig. 3, for $\gamma_{\phi, 2}=0.1, \Gamma=0$, and $d_{S} / \xi_{S}=0.5$, the gap of the DOS would be reduced but still finite (not shown). In these conditions, using a small $\Gamma$ can trigger the gap suppression as shown by the black full line in Fig. 4, right panel. In the $G_{\phi, 1} \neq 0$ case, the effects of $G_{\phi, 2}$ on a thin $S$ remain qualitatively similar, in particular, the gap reduction in the DOS still occurs, combined with the $G_{\phi, 1}$-induced spin splitting. Figure 2, left 


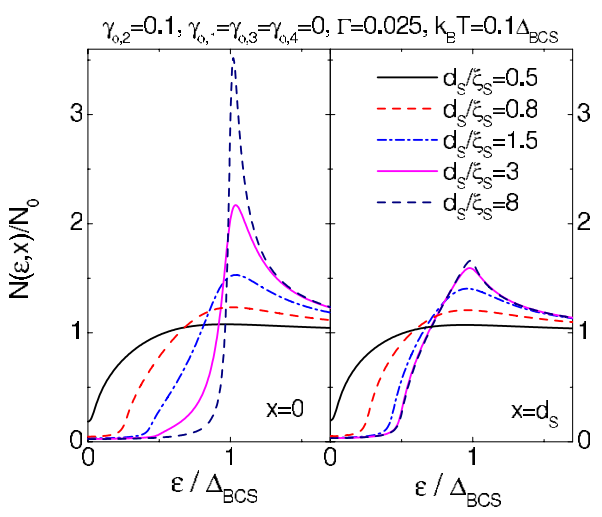

FIG. 4. (Color online) Density of states in the $S$ layer contacted to a $F I$, for $x=0$ (left panel) and $x=d_{S}$ (right panel), and different values of $d_{S} / \xi_{S}$. In all cases, we have used $\gamma_{\phi, 2}=0.1, \gamma_{\phi, 1}=\gamma_{\phi, 3}$ $=\gamma_{\phi, 4}=0, \Gamma=0.025$, and $k_{B} T=0.1 \Delta_{\mathrm{BCS}}$.

panel compares a case with $G_{\phi, 2}=0$ and a large $\Gamma$ (black dashed line) with a case with $G_{\phi, 2} \neq 0$ and a small $\Gamma$ (blue full line). The two cases can easily be discriminated due to the different curvatures in the DOS. Importantly, the analogy between a paramagnetic impurity term and the $G_{\phi, 2}$ term is not complete since $G_{\phi, 2}$ occurs in the BCIGF whereas paramagnetic impurities would contribute directly to the Usadel equation. This discrepancy is revealed by the dependence of the DOS on $d_{S}$. Figure 4 presents the DOS at the left and right side of the $S$ layer for different values of $d_{S}$ and $G_{\phi, 1}$ $=0$. We obtain a strong dependence of the $G_{\phi, 2}$ DOS widening on $x$ and $d_{S}$. First, for $d_{S} / \xi_{S}=0.5$, the DOS at the left and the right sides of $S$ (left and right panels) are almost identical with a suppressed gap for the parameters we consider. When $d_{S}$ increases, the gap reappears in the DOS. For $d_{S} \gg \xi_{S}$, the DOS at the left side of $S$ tends to the bulk BCS DOS, with no effect of $G_{\phi, 2}$, whereas the DOS at the right side of $S$ still has a reduced gap. In this limit, one can check that the reduction of the gap occurs for a slab of $S$ of thickness $\sim \xi_{S}$ near the $S / F I$ interface. In contrast, paramagnetic impurities would affect the bulk of $S$. Let us now consider the case $G_{\phi, 1} \neq 0$ and $d_{S}$ large. In this case, Ref. 25 has shown that the $G_{\phi, 1}$-induced spin splitting of the DOS can persist in a slab of $S$ of thickness $\sim \xi_{S}$ near the $S / F I$ interface. The right panel of Fig. 2 shows an example of DOS at $x=d_{S}$ for $d_{S}=3 \xi_{S}$, in the case $G_{\phi, 1} \neq 0, G_{\phi, 2}=0$, and a large $\Gamma$ (black dashed line), and in the case $G_{\phi, 1} \neq 0, G_{\phi, 2} \neq 0$, and a small $\Gamma$ (blue full line). In the first case, the $G_{\phi, 1}$-induced spin splitting of the DOS is not visible anymore because $H_{\text {eff }}$ scales with $1 / d_{S}$ and thus becomes too small compared to the large value of $\Gamma$ used. In the second case, the double-gap splitting is still slightly visible as a cusp in the DOS curve because the $G_{\phi, 2}$ DOS widening also decreases with $d_{S}$. The effects of the $G_{\phi, 3}$ and $G_{\phi, 4}$ terms in the general case will be presented elsewhere. Before concluding, we note that in circuits enclosing several FI with noncollinear magnetizations and BCS superconductors, it has been found that the $G_{\phi, 1}$ term can induce spin-precession effects which lead to superconducting correlations between equal spins. ${ }^{30}$

\section{CONCLUSION}

To model the behavior of electronic hybrid circuits, a proper description of the contacts between the different ma- terials is crucial. In this paper, we have derived general boundary conditions relating isotropic Green's functions at both sides of the interface between two diffusive materials [Eqs. (39)-(41)]. These BCIGF are valid for a circuit enclosing superconductors, normal metals, and ferromagnets, in a possibly noncollinear geometry. In general, they require the knowledge of the full contact scattering matrix, an information usually not available for realistic interfaces. However, we have shown that in the limit of a specular tunnel contact with weakly spin-dependent scattering properties, the BCIGF can be expressed in terms of a few parameters, i.e., the tunnel conductance $G_{T}$ of the contact, a parameter $G_{M R}$ which accounts for the spin dependence of the contact scattering probabilities, and four parameters $G_{\phi}^{L(R)}$ and $G_{\chi}^{L(R)}$ which are finite when the contact exhibits a SDIPS [Eqs. (61) and (62)]. In the case of a contact with a FI side, we could simplify the BCIGF for a stronger SDIPS [Eq. (73)]. We believe that the various spin-dependent BCIGF derived in this paper represent a solid basis for further developments on superconducting hybrid circuits.

\section{ACKNOWLEDGMENTS}

We acknowledge discussions with A. Brataas, B. Douçot, T. Kontos, J. P. Morten, and S. Sadjina. This work was financially supported by the DFG under Grants No. SFB 513 and No. SFB 767, and the Landesstiftung BadenWürttemberg (WB). We acknowledge the hospitality of the Workshop "Spin and Charge Flow in Nanostructures" at the CAS, Oslo.

\section{APPENDIX A: SCATTERING DESCRIPTION OF A SPECULAR AND SPIN-CONSERVING CONTACT}

\section{Structure of the electronic scattering matrix}

In this section, we assume that the transverse channel index $n$ and the spin index $\sigma \in\{\uparrow, \downarrow\}$, corresponding to spin components along $\vec{Z}$, are conserved when electrons cross the potential barrier $\bar{V}_{b}$ between the two ballistic zones. In this case, the electronic scattering matrix $\mathcal{S}^{e}$ is diagonal in the (transverse channel) $\otimes$ spin subspace. The scattering submatrix associated to electrons with spins $\sigma$ of the $n$th transverse channel writes

$$
\mathcal{S}_{n \sigma}^{e}=\left[\begin{array}{ll}
r_{L, n \sigma} & t_{R, n \sigma} \\
t_{L, n \sigma} & r_{R, n \sigma}
\end{array}\right] \text {. }
$$

Here, $r_{L(R), n \sigma}$ denotes the reflection amplitude at side $L(R)$ of the barrier and $t_{R(L), n \sigma}$ the transmission amplitude from side $R(L)$ to side $L(R)$. Flux conservation imposes, for $\sigma$ $\in\{\uparrow, \downarrow\}, \quad \Sigma_{Q \in\{L, R\}}\left[\arg \left(r_{Q, n \sigma}\right)-\arg \left(t_{Q, n \sigma}\right)\right]=\pi[2 \pi] \quad$ and 1 $-\left|r_{Q, n \sigma}\right|^{2}=\left|t_{Q, n \sigma}\right|^{2}=T_{n \sigma}$. In addition, spin conservation along $\vec{Z}$ allows to map the scattering description of each spin component $\sigma$ onto a spinless problem. Time reversal symmetry in each of these spinless problems implies $\arg \left(t_{L, n \sigma}\right)$ $=\arg \left(t_{R, n \sigma}\right)$. Therefore, one can use, without any loss of generality 


$$
\mathcal{S}_{n \sigma}^{e}=\left[\begin{array}{cc}
\sqrt{1-T_{n \sigma}} e^{i \varphi_{n \sigma}^{L}} & i \sqrt{T_{n \sigma}} e^{i\left(\varphi_{n \sigma}^{L}+\varphi_{n \sigma}^{R}\right) / 2} \\
i \sqrt{T_{n \sigma}} e^{i\left(\varphi_{n \sigma}^{L}+\varphi_{n \sigma}^{R}\right) / 2} & \sqrt{1-T_{n \sigma}} e^{i \varphi_{n \sigma}^{R}}
\end{array}\right]
$$

with $\varphi_{n \sigma}^{L(R)}=\arg \left(r_{L(R), n \sigma}\right)$. The matrix $\mathcal{S}_{n \sigma}^{e}$ is entirely determined by $T_{n \sigma}, \varphi_{n \sigma}^{L}$, and $\varphi_{n \sigma}^{R}$. In this paper, we use the parametrization $T_{n \sigma}=T_{n}\left(1+\sigma P_{n}\right)$ and $\varphi_{n \sigma}^{L(R)}=\varphi_{n}^{L(R)}+\sigma\left(d \varphi_{n}^{L(R)} / 2\right)$ [Eqs. (42) and (43)].

\section{Expression of the transfer matrix with scattering parameters}

In this section, we assume that the transmission amplitudes $t_{L(R), n \sigma}$ are finite. With the hypotheses made in Appendix A 1 , the electronic transfer matrix $\mathcal{M}^{e}$ is also diagonal in the (transverse channel) $\otimes$ spin subspace. In the propagation direction subspace, the submatrix $\mathcal{M}_{n \sigma}^{e}$ has elements ${ }^{51}$

$$
\begin{gathered}
\mathcal{M}_{n \sigma,+,+}^{e}=\left(t_{L, n \sigma}^{\dagger}\right)^{-1}, \\
\mathcal{M}_{n \sigma,+,-}^{e}=r_{R, n \sigma}\left(t_{R, n \sigma}\right)^{-1}, \\
\mathcal{M}_{n \sigma,-,+}^{e}=-\left(t_{R, n \sigma}\right)^{-1} r_{L, n \sigma}, \\
\mathcal{M}_{n \sigma,-,-}^{e}=\left(t_{R, n \sigma}\right)^{-1} .
\end{gathered}
$$

We have used above $+/-$ to denote the right/left-going propagation direction. Using Eqs. (23) and (A2)-(A5) and the parametrization introduced in Appendix A 1, one can obtain and expression for the matrix $\bar{M}$ in terms of $T_{n}, P_{n}$, $\varphi_{n}^{L(R)}$, and $d \varphi_{n}^{L(R)}$. At first order in $P_{n}$ and $d \varphi_{n}^{L(R)}$, this leads to the expressions (44)-(48).

\section{APPENDIX B: CALCULATION OF $\check{I}_{L}^{(1)}(\varepsilon)$ FOR A $S / F$ CONTACT}

In this section, we give details on the calculation of the contribution $\breve{I}_{L}^{(1)}(\varepsilon)$ to the matrix current $\breve{I}_{L}(\varepsilon)$ to first order in $\delta \bar{X}$. Using Eq. (54), one can rewrite Eq. (59) as

$$
\begin{aligned}
\check{I}_{L}^{(1)}(\varepsilon)= & -4 G_{q} \operatorname{Tr}_{n}\left\{\hat{T}_{0}\left(4+\hat{T}_{0}\left[\left\{\check{G}_{L}, \check{G}_{R}\right\}-2\right]\right)^{-1}\right. \\
& \left.\times \operatorname{Tr}_{s}[\widetilde{W}] \hat{T}_{0}\left(4+\hat{T}_{0}\left[\left\{\check{G}_{L}, \check{G}_{R}\right\}-2\right]\right)^{-1}\right\} .
\end{aligned}
$$

The central term

$$
\widetilde{W}=\left(\check{G}_{R} \check{G}_{L}+\hat{Q}_{0}^{-1}\right) \delta \widetilde{V}\left(\check{G}_{R} \check{G}_{L}+\hat{Q}_{0}^{-1}\right)\left(\mathbf{1}+\hat{\Sigma}_{3} \check{G}_{L}\right)
$$

of this expression can be decomposed as $\widetilde{W}=\Sigma_{j=1}^{4} \widetilde{W}_{j}$ with

$$
\begin{aligned}
\widetilde{W}_{1}= & \hat{Q}_{0} \delta \bar{X} \check{G}_{R} \check{G}_{L}+\check{G}_{R} \delta \bar{X}^{\dagger} \hat{Q}_{0} \check{G}_{L}+\check{G}_{L} \check{G}_{R} \delta \bar{X} \hat{Q}_{0}^{-1} \\
& +\check{G}_{L} \hat{Q}_{0}^{-1} \delta \bar{X}^{\dagger} \check{G}_{R}, \\
\widetilde{W}_{2}= & \hat{Q}_{0} \delta \bar{X} \hat{Q}_{0}^{-1}+\check{G}_{R} \delta \bar{X}^{\dagger} \check{G}_{R}+\check{G}_{L} \check{G}_{R} \delta \bar{X} \check{G}_{R} \check{G}_{L} \\
& +\check{G}_{L} \hat{Q}_{0}^{-1} \delta \bar{X}^{\dagger} \hat{Q}_{0} \check{G}_{L},
\end{aligned}
$$

and $\widetilde{W}_{3(4)}=\widetilde{W}_{1(2)} \hat{\Sigma}_{3} \check{G}_{L}$. We now develop the trace over the propagation direction index $s$ in Eq. (B1), using expressions
(46), (56), and (57), and keeping in mind that $\check{G}_{L}$ and $\check{G}_{R}$ have no structure in the $\underline{\mathcal{E}}$ subspace. We find $\operatorname{Tr}_{s}(\delta \bar{X})$ $=\operatorname{Tr}_{s}\left(\delta \bar{X}^{\dagger}\right)=\operatorname{Tr}_{s}\left(\hat{Q}_{0} \delta \bar{X} \hat{Q}_{0}^{-1}\right)=\operatorname{Tr}_{s}\left(\hat{Q}_{0}^{-1} \delta \bar{X}^{\dagger} \hat{Q}_{0}\right)=0 \quad$ so $\quad$ that $\operatorname{Tr}_{s}\left(\widetilde{W}_{2}\right)=0$. Due to Eqs. (56) and (57), we find $\operatorname{Tr}_{s}\left(\hat{Q}_{0} \hat{\Sigma}_{3}\right)$ $=\operatorname{Tr}_{s}\left(\hat{Q}_{0}^{-1} \hat{\Sigma}_{3}\right)=0$. Hence, $\delta \bar{X}_{++}$and the diagonal elements of $\hat{Q}_{0}$ and $\hat{Q}_{0}^{-1}$ do not contribute to $\operatorname{Tr}_{s}\left(\tilde{W}_{1}\right)$. In contrast, the development of $\operatorname{Tr}_{s}\left(\widetilde{W}_{3(4)}\right)$ involves both $\delta \bar{X}_{++}$and $\delta \bar{X}_{+-}$. We finally obtain

$$
\begin{gathered}
\operatorname{Tr}_{s}\left[\tilde{W}_{1}\right]=\left[\left\{A, \check{G}_{R}\right\}, \check{G}_{L}\right], \\
\operatorname{Tr}_{s}\left[\widetilde{W}_{3}\right]=\left[\left[C, \check{G}_{R}\right] \check{G}_{L}, \check{G}_{L}\right], \\
\operatorname{Tr}_{s}\left[\widetilde{W}_{4}\right]=2\left[B-\check{G}_{R}\left[\delta \bar{X}_{++}, \check{G}_{R}\right], \check{G}_{L}\right]
\end{gathered}
$$

with

$$
\begin{gathered}
A[F]=\hat{Q}_{0,+-} \delta \bar{X}_{+-}^{*}+[-] \hat{Q}_{0,++} \delta \bar{X}_{+-} \\
C=2 \hat{Q}_{0,++} \delta \bar{X}_{++}+F \\
B=\left(\hat{Q}_{0,++}^{2}+\hat{Q}_{0,+} \hat{Q}_{0,-+}\right) \delta \bar{X}_{++}+\hat{Q}_{0,++} F-\delta \bar{X}_{++}
\end{gathered}
$$

Expressing $\hat{Q}_{0}$ and $\delta \bar{X}$ in terms of the scattering parameters $T_{n}, P_{n}, \varphi_{n}^{L(R)}$, and $d \varphi_{n}^{L(R)}$ [see Eqs. (46)-(48) and (56)], and developing the trace on transverse channels in Eq. (B1), we obtain the expression (60) for $\check{I}_{L}^{(1)}(\varepsilon)$.

\section{APPENDIX C: GENERAL BOUNDARY CONDITIONS IN THE NORMAL-STATE LIMIT}

When there are no superconducting correlations in the circuit, the isotropic Green's functions $\check{G}_{L(R)}$ write, in the Keldysh space

$$
\check{G}_{L(R)}=\left[\begin{array}{cc}
\check{\tau}_{3} & \check{K}_{L(R)} \\
0 & -\check{\tau}_{3}
\end{array}\right] .
$$

In this limit, the elements $\widetilde{D}_{L}^{-1}$ and $\widetilde{D}_{R}^{-1}$ appearing in the general BCIGF Eqs. (39) and (40) take a simple form. For instance, one finds, in the Keldysh space

$$
\tilde{D}_{L}^{-1}=\left[\begin{array}{cc}
\bar{N}_{L} & -\check{\tau}_{3} \bar{N}_{L}\left(\bar{M}^{\dagger} \check{K}_{R} \bar{M}-\check{K}_{L} \bar{M}^{\dagger} \bar{M}\right) \bar{N}_{L} \\
0 & \bar{N}_{L}
\end{array}\right]
$$

with $\bar{N}_{L}=\left(1+\bar{M}^{\dagger} \bar{M}\right)^{-1}$. A similar expression can be obtained for $\widetilde{D}_{R}^{-1}$ by replacing $\bar{M}$ by $\bar{M}^{-1}$ and $\check{K}_{L[R]}$ by $\check{K}_{R[L]}$. For comparison with Secs. VII and VIII, we specialize to the case of a specular contact conserving spins along the interface magnetization. Equations (39) and (40) give, for the Keldysh electronic component of the matrix currents

$$
\check{I}_{L}^{K, e}(\varepsilon)=2 G_{q} \operatorname{Tr}_{n}\left[-t_{R} \check{K}_{R}^{e} t_{R}^{\dagger}+\check{K}_{L}^{e}-r_{L} \check{K}_{L}^{e} r_{L}^{\dagger}\right]
$$

and

$$
\check{I}_{R}^{K, e}(\varepsilon)=2 G_{q} \operatorname{Tr}_{n}\left[t_{L} \check{K}_{L}^{e} t_{L}^{\dagger}-\check{K}_{R}^{e}+r_{R} \check{K}_{R}^{e} r_{R}^{\dagger}\right] .
$$

Assuming that the contact is magnetized along $\vec{Z}$, we obtain 


$$
\begin{aligned}
\check{I}_{L(R)}^{K, e}(\varepsilon) / 2= & \left(\left[G_{T} / 2\right]+G_{M R}\right) \check{u}_{\uparrow}\left[\check{K}_{L}^{e, \uparrow, \uparrow}-\check{K}_{R}^{e, \uparrow, \uparrow}\right] \check{u}_{\uparrow} \\
& +\left(\left[G_{T} / 2\right]-G_{M R}\right) \check{u}_{\downarrow}\left[\check{K}_{L}^{e, \downarrow, \downarrow}-\check{K}_{R}^{e, \downarrow \downarrow \downarrow}\right] \check{u}_{\downarrow} \\
& \mp G_{m i x}^{t} \check{u}_{\uparrow} \check{K}_{R(L)}^{e, \uparrow} \check{u}_{\downarrow} \mp\left(G_{m i x}^{t}\right)^{*} \check{u}_{\downarrow} \check{K}_{R(L)}^{e, \downarrow \uparrow} \check{u}_{\uparrow} \\
& \pm G_{m i x}^{L(R), r} \check{u}_{\uparrow} \check{K}_{L(R)}^{e, \uparrow}, \check{u}_{\downarrow} \pm\left(G_{m i x}^{L(R), r}\right)^{*} \check{u}_{\downarrow} \check{K}_{L(R)}^{e, \downarrow \uparrow} \check{u}_{\uparrow}
\end{aligned}
$$

with $\check{u}_{\uparrow(\downarrow)}=1 \pm\left(\check{\sigma}_{Z} / 2\right)$,

$$
G_{m i x}^{t}=G_{q} \sum_{n} t_{L, n \downarrow}^{*} t_{L, n \uparrow},
$$

and

$$
G_{\text {mix }}^{L(R), r}=G_{q} \sum_{n}\left(1-r_{L(R), n \downarrow}^{*} r_{L(R), n \uparrow}\right) .
$$

We have checked that in the normal-state limit, Eq. (71) leads to Eq. (C2) with $t_{R}=0$. Equations $(\mathrm{C} 2)-(\mathrm{C} 4)$ are in agreement with the normal-state BCIGF presented, e.g., in Eq. (2) of Ref. 36, up to a prefactor which corresponds to our conventions. ${ }^{74}$ Importantly, the derivation of these equations requires no particular assumptions on the values of $t_{L(R), n \sigma}$ and $r_{L(R), n \sigma}$. In the normal-state limit, a strong spin relaxation is often assumed in $F$ so that the $G_{\text {mix }}^{t}$ term is disregarded [see, e.g., Eq. (5) of Ref. 36]. When the circuit includes superconducting elements, the expressions of $\widetilde{D}_{L}^{-1}$ and $\widetilde{D}_{R}^{-1}$ involve, e.g., factors $\left(1+\check{G}_{L}^{a(r)} \bar{M}^{\dagger} \breve{G}_{R}^{a(r)} \bar{M}\right)^{-1}$ instead of $\bar{N}_{L}$. This is why the superconducting BCIGF are difficult to simplify in the general case.

\section{APPENDIX D: EQUILIBRIUM BOUNDARY CONDITIONS IN THE CASE OF SUPERCONDUCTING CORRELATIONS BETWEEN OPPOSITE SPINS ONLY}

This appendix presents the boundary conditions obeyed by the retarded part of the isotropic Green's functions, in a case where there are superconducting correlations between opposite spins only. This situation occurs, e.g., when all the ferromagnetic elements of the circuit are magnetized in collinear directions. For simplicity, we assume that no phase gradient is present in the system. The conventions chosen in Sec. II give, inside conductor $Q$

$$
\check{G}_{Q}^{r}=\left[\begin{array}{cccc}
\cos \left(\Lambda_{\uparrow}^{Q}\right) & 0 & 0 & \sin \left(\Lambda_{\uparrow}^{Q}\right) \\
0 & \cos \left(\Lambda_{\downarrow}^{Q}\right) & \sin \left(\Lambda_{\downarrow}^{Q}\right) & 0 \\
0 & \sin \left(\Lambda_{\downarrow}^{Q}\right) & -\cos \left(\Lambda_{\downarrow}^{Q}\right) & 0 \\
\sin \left(\Lambda_{\uparrow}^{Q}\right) & 0 & 0 & -\cos \left(\Lambda_{\uparrow}^{Q}\right)
\end{array}\right]
$$

with $\Lambda_{\uparrow}^{Q}=\Lambda_{\uparrow}^{Q}$ in the spin-degenerate case. For a metallic contact, using Eqs. (41), (61), and (62), one obtains

$$
\begin{aligned}
-\frac{A}{\rho_{L}} \frac{\partial \Lambda_{\sigma}^{L}}{\partial z}= & G_{T} \sin \left(\Lambda_{\sigma}^{L}-\Lambda_{\sigma}^{R}\right)+i G_{\phi}^{L} \sigma \sin \left(\Lambda_{\sigma}^{L}\right) \\
& +2 i \sin \left(\Lambda_{\sigma}^{R}\right) \sigma\left[G_{\chi}^{R}-G_{\chi}^{L} \cos \left(\Lambda_{\sigma}^{L}-\Lambda_{\sigma}^{R}\right)\right]
\end{aligned}
$$

and

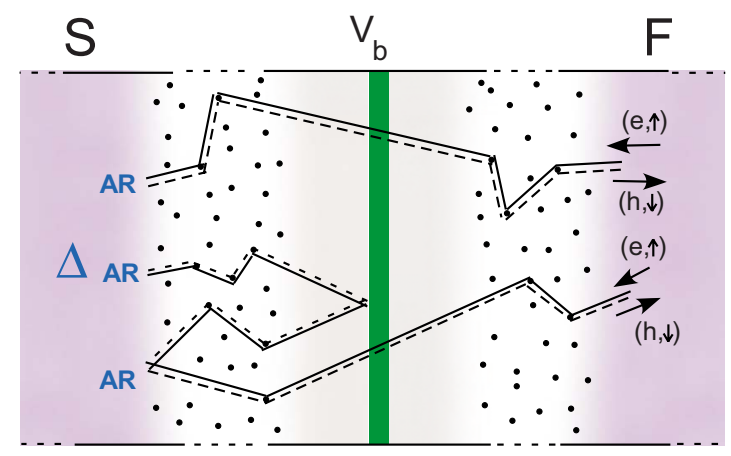

FIG. 5. (Color online) Scheme representing two particular types of Andreev reflection processes which can occur on a $S / F$ interface modeled like in Fig. 1. The ballistic, isotropization, and diffusive zones of $S$ and $F$ are represented by gray, dotted, and purple areas, respectively. Full (dashed) lines represent trajectories of electrons (holes) from the $\uparrow(\downarrow)$ spin band. The superconducting gap $\Delta$ is taken into account in the diffusive part of $S$ only so that one can consider that Andreev reflections occur at the interface between the diffusive and isotropization zones of $S$. The upper part of the scheme represents and electron incident from the $F$ side, which is transmitted by the barrier $V_{b}$ as an electron, Andreev reflected on the diffusive part of $S$ as a hole, and transmitted again by $V_{b}$ as a hole, before joining the diffusive part of $F$ again. The probability associated to this process is proportional to $T_{n}^{2}\left(1-P_{n}^{2}\right)$. The lower part of the scheme represents a more complicated trajectory which also involves two reflections on $V_{b}$. The joint probability of these reflections is $\left(1-T_{n}\right)^{2}-T_{n}^{2} P_{n}^{2}$

$$
\begin{aligned}
-\frac{A}{\rho_{R}} \frac{\partial \Lambda_{\sigma}^{R}}{\partial z}= & G_{T} \sin \left(\Lambda_{\sigma}^{L}-\Lambda_{\sigma}^{R}\right)-i G_{\phi}^{R} \sigma \sin \left(\Lambda_{\sigma}^{R}\right) \\
& -2 i \sigma \sin \left(\Lambda_{\sigma}^{L}\right)\left[G_{\chi}^{L}-G_{\chi}^{R} \cos \left(\Lambda_{\sigma}^{R}-\Lambda_{\sigma}^{L}\right)\right] .
\end{aligned}
$$

Interestingly, the $G_{M R}$ term vanishes from Eqs. (D2) and (D3) so that the tunnel-rate polarization $P_{n}$ does not contribute to the equilibrium BCIGF (we have checked that this property remains true when phase gradients occur in the system). This result may seem surprising since Andreev reflections, which modify the equilibrium density of states in a superconducting hybrid system, are suppressed when $P_{n}$ is strong. ${ }^{75}$ However, one should keep in mind that an Andreev reflection process on the $L / R$ interface involves together the transmission (or reflection) of an electron and a hole from opposite-spin bands through the $\bar{V}_{b}$ barrier (see Fig. 5). These two processes have a joint probability which involves $P_{n}^{2}$. In contrast, single quasiparticle processes, whose probabilities involve $P_{n}$ at first order, do not matter at equilibrium. We conclude that $P_{n}$ vanishes from the equilibrium BCIGF Eqs. (D2) and (D3) because we have derived these equations at first order in $P_{n}$. Note that even in this limit, $P_{n}$ does not vanish from the boundary conditions obeyed by the Keldysh part of the isotropic Green's functions [see, e.g., Eq. (C4)].

For completeness, we mention that in the case of a contact with a FI side, using Eqs. (41) and (73), one obtains 


$$
\begin{aligned}
-\frac{A}{\rho_{L}} \frac{\partial \Lambda_{\sigma}^{L}}{\partial z}= & i G_{\phi, 1} \sigma \sin \left(\Lambda_{\sigma}^{L}\right)+G_{\phi, 2}^{L} \sin \left(2 \Lambda_{\sigma}^{L}\right) \\
& +i G_{\phi, 3}^{L} \sigma \sin \left(3 \Lambda_{\sigma}^{L}\right)+G_{\phi, 4}^{L} \sin \left(4 \Lambda_{\sigma}^{L}\right) .
\end{aligned}
$$

\section{APPENDIX E: USADEL EQUATIONS}

For completeness, we mention that the Usadel equations corresponding to Eqs. (6) and (7) write, inside conductor $Q$ (see, e.g., Ref. 40)

$$
\hbar D_{Q} \frac{\partial}{\partial z}\left(\check{G} \frac{\partial}{\partial z} \check{G}\right)=\left[-i \varepsilon \check{\tau}_{3}+\check{\Delta}(z)+i E_{e x}(z) \check{\sigma}_{Z}, \check{G}\right] .
$$

The gap matrix $\check{\Delta}$ has a structure in the Nambu-spin subspace only, i.e., with our conventions

$$
\check{\Delta}(z)=\left[\begin{array}{lll} 
& & \Delta(z) \\
& \Delta(z) & \\
\Delta(z)^{*} & &
\end{array}\right] .
$$

Using the angular parametrization of Appendix D, Eq. (E1) leads to

$$
\frac{\hbar D_{Q}}{2} \frac{\partial^{2} \Lambda_{\sigma}}{\partial z^{2}}=\left[-i \varepsilon+i \sigma E_{e x}(z)\right] \sin \left(\Lambda_{\sigma}\right)-\Delta(z) \cos \left(\Lambda_{\sigma}\right) .
$$

${ }^{1}$ M. N. Baibich, J. M. Broto, A. Fert, F. Nguyen Van Dau, F. Petroff, P. Etienne, G. Creuzet, A. Friederich, and J. Chazelas, Phys. Rev. Lett. 61, 2472 (1988); G. Binasch, P. Grünberg, F. Saurenbach, and W. Zinn, Phys. Rev. B 39, 4828 (1989).

${ }^{2}$ J. Slonczewski, J. Magn. Magn. Mater. 159, L1 (1996); L. Berger, Phys. Rev. B 54, 9353 (1996); E. B. Myers, D. C. Ralph, J. A. Katine, R. N. Louie, and R. A. Buhrman, Science 285, 867 (1999); J. A. Katine, F. J. Albert, R. A. Buhrman, E. B. Myers, and D. C. Ralph, Phys. Rev. Lett. 84, 3149 (2000); X. Waintal, E. B. Myers, P. W. Brouwer, and D. C. Ralph, Phys. Rev. B 62, 12317 (2000).

${ }^{3}$ D. C. Ralph and M. D. Stiles, J. Magn. Magn. Mater. 320, 1190 (2008).

${ }^{4}$ A. F. Andreev, Sov. Phys. JETP 19, 1228 (1964).

${ }^{5}$ W. L. McMillan, Phys. Rev. 175, 537 (1968).

${ }^{6}$ A. I. Buzdin, L. N. Bulaevskii, and S. V. Panyukov, JETP Lett. 35, 178 (1982).

${ }^{7}$ T. Kontos, M. Aprili, J. Lesueur, and X. Grison, Phys. Rev. Lett. 86, 304 (2001)

${ }^{8}$ V. V. Ryazanov, V. A. Oboznov, A. Yu. Rusanov, A. V. Veretennikov, A. A. Golubov, and J. Aarts, Phys. Rev. Lett. 86, 2427 (2001).

${ }^{9}$ T. Kontos, M. Aprili, J. Lesueur, F. Genêt, B. Stephanidis, and R. Boursier, Phys. Rev. Lett. 89, 137007 (2002).

${ }^{10}$ W. Guichard, M. Aprili, O. Bourgeois, T. Kontos, J. Lesueur, and P. Gandit, Phys. Rev. Lett. 90, 167001 (2003).

${ }^{11}$ L. B. Ioffe, V. B. Geshkenbein, M. V. Feigel'man, A. L. Fauchère, and G. Blatter, Nature (London) 398, 679 (1999).

${ }^{12}$ T. Yamashita, K. Tanikawa, S. Takahashi, and S. Maekawa, Phys. Rev. Lett. 95, 097001 (2005).

${ }^{13}$ F. S. Bergeret, A. F. Volkov, and K. B. Efetov, Rev. Mod. Phys. 77, 1321 (2005).

${ }^{14}$ A. A. Golubov, M. Yu. Kupryanov, and E. Il'ichev, Rev. Mod. Phys. 76, 411 (2004).

${ }^{15}$ A. I. Buzdin, Rev. Mod. Phys. 77, 935 (2005).

${ }^{16}$ K. D. Usadel, Phys. Rev. Lett. 25, 507 (1970).

${ }^{17}$ M. Yu. Kuprianov and V. F. Lukichev, Sov. Phys. JETP 67, 1163 (1988).

${ }^{18}$ A. Millis, D. Rainer, and J. A. Sauls, Phys. Rev. B 38, 4504 (1988).
${ }^{19}$ T. Tokuyasu, J. A. Sauls, and D. Rainer, Phys. Rev. B 38, 8823 (1988).

${ }^{20}$ M. Fogelström, Phys. Rev. B 62, 11812 (2000); J. C. Cuevas and M. Fogelström, ibid. 64, 104502 (2001); J. Kopu, M. Eschrig, J. C. Cuevas, and M. Fogelström, ibid. 69, 094501 (2004).

${ }^{21}$ E. Zhao, T. Löfwander, and J. A. Sauls, Phys. Rev. B 70, 134510 (2004).

${ }^{22}$ D. Huertas-Hernando, Yu. V. Nazarov, and W. Belzig, Phys. Rev. Lett. 88, 047003 (2002).

${ }^{23}$ D. Huertas-Hernando and Yu. V. Nazarov, Eur. Phys. J. B 44, 373 (2005)

${ }^{24}$ A. Cottet, Phys. Rev. B 76, 224505 (2007).

${ }^{25}$ A. Cottet and J. Linder, Phys. Rev. B 79, 054518 (2009).

${ }^{26}$ A. Cottet and W. Belzig, Phys. Rev. B 72, 180503(R) (2005).

${ }^{27}$ J. Linder, T. Yokoyama, and A. Sudbø, Phys. Rev. B 79, 054523 (2009).

${ }^{28}$ J. P. Morten, D. Huertas-Hernando, A. Brataas, and W. Belzig, EPL 81, 40002 (2008).

${ }^{29}$ J. P. Morten, D. Huertas-Hernando, W. Belzig, and A. Brataas, Phys. Rev. B 78, 224515 (2008).

${ }^{30}$ V. Braude and Yu. V. Nazarov, Phys. Rev. Lett. 98, 077003 (2007).

${ }^{31}$ J. Linder, T. Yokoyama, A. Sudbø, and M. Eschrig, Phys. Rev. Lett. 102, 107008 (2009).

${ }^{32}$ A. Di Lorenzo and Y. V. Nazarov, Phys. Rev. Lett. 94, 210601 (2005).

${ }^{33}$ G. Falci, D. Feinberg, and F. W. J. Hekking, Europhys. Lett. 54, 255 (2001).

${ }^{34}$ D. Sánchez, R. López, P. Samuelsson, and M. Büttiker, Phys. Rev. B 68, 214501 (2003).

${ }^{35}$ A. Brataas, Yu. V. Nazarov, and G. E. W. Bauer, Phys. Rev. Lett. 84, 2481 (2000).

${ }^{36}$ A. Brataas, Yu. V. Nazarov, and G. E. W. Bauer, Eur. Phys. J. B 22, 99 (2001).

${ }^{37}$ Yu. V. Nazarov, Superlattices Microstruct. 25, 1221 (1999).

${ }^{38}$ Yu. V. Nazarov, Ann. Phys. (Leipzig) 8, SI-193 (1999).

${ }^{39}$ W. Belzig and Yu. V. Nazarov, Phys. Rev. Lett. 87, 067006 (2001); 87, 197006 (2001).

${ }^{40}$ W. Belzig, F. K. Wilhelm, C. Bruder, G. Schön, and A. Zaikin, Superlattices Microstruct. 25, 1251 (1999). 
${ }^{41}$ J. W. A. Robinson, S. Piano, G. Burnell, C. Bell, and M. G. Blamire, Phys. Rev. Lett. 97, 177003 (2006).

${ }^{42}$ J. Kim, J. H. Kwon, K. Char, H. Doh, and H.-Y. Choi, Phys. Rev. B 72, 014518 (2005).

${ }^{43}$ I. C. Moraru, W. P. Pratt, Jr., and Norman O. Birge, Phys. Rev. Lett. 96, 037004 (2006).

${ }^{44}$ S. Guéron, H. Pothier, Norman O. Birge, D. Esteve, and M. H. Devoret, Phys. Rev. Lett. 77, 3025 (1996).

${ }^{45}$ J. C. Cuevas and W. Belzig, Phys. Rev. Lett. 91, 187001 (2003); Phys. Rev. B 70, 214512 (2004).

${ }^{46}$ I. Snyman and Yu. V. Nazarov, Phys. Rev. B 77, 165118 (2008).

${ }^{47}$ A. V. Zaitsev, Sov. Phys. JETP 59, 1015 (1984).

${ }^{48}$ A. L. Shelankov, J. Low Temp. Phys. 60, 29 (1985).

${ }^{49} \mathrm{We}$ use the convention $\operatorname{sgn}(0)=0$ so that the expression of $\mathrm{G}\left(\vec{r}, \vec{r}^{\prime}, \varepsilon\right)$ obtained by combining Eqs. (11) and (12) is continuous at $\vec{r}=\vec{r}^{\prime}$, in agreement with the definitions Eqs. (1)-(5).

${ }^{50}$ Ya. M. Blanter and M. Büttiker, Phys. Rep. 336, 1 (2000).

${ }^{51}$ A. D. Stone, P. A. Mello, K. A. Muttalib, and J.-L. Pichard, in Mesoscopic Phenomena in Solids, edited by B. L. Altshuler, P. A. Lee, and R. A. Webb (North-Holland, Amsterdam, 1991).

${ }^{52}$ Equations (28)-(33) confirm that the size $d_{L(R)}$ of the isotropization zones just needs to be on the order of a few $\ell_{\mathrm{e}}^{L(R)}$ due to the exponential factors $\exp \left[-z / 2 \overline{\bar{v}}_{Q} \tau_{Q}\right]$. In other terms, only a few collisions are necessary to isotropize the Green's functions.

${ }^{53}$ A. A. Abrikosov, L. P. Gor'kov, and I. E. Dzialoshinskii, Methods of Quantum Field Theory in Statistical Physics (Dover, New York, 1963).

${ }^{54}$ The quasiclassical isotropic average of $\mathrm{G}\left(\vec{r}, \vec{r}^{\prime}, \varepsilon\right)$ can be calculated as $\check{G}_{q c i}(\vec{R}, \varepsilon)=i \mathrm{G}\left(\vec{r}=\vec{R}, \vec{r}^{\prime}=\vec{R}, \varepsilon\right) / \pi \nu_{0}$ [see Eq. (9)]. From Eq. (34), for $z \rightarrow \mp d_{L(R)}$, one has $\tilde{\mathcal{G}}_{\text {diff }}(z, z, \varepsilon)=-i \pi \check{G}_{L(R)}$. Inserting this expression into Eq. (11) leads to $\check{G}_{q c i}(\vec{R}, \varepsilon)=\check{G}_{L(R)} \mathcal{C} / \nu_{0}$ with $\mathcal{C}=\Sigma_{n}\left|\chi_{n}(\vec{\rho})\right|^{2} / \pi \hbar v_{n}$. We note that if $t_{L(R), n \sigma}=1$ for any $n$ and $\sigma$, the normal-state electronic density of states at energy $\epsilon$ for a given spin direction writes (Ref. 50) $N_{\sigma}(\vec{R}, \varepsilon)=\mathcal{C}$ but also $N_{\sigma}(\vec{R}, \varepsilon)=\nu_{0}$. The resulting identity $\mathcal{C}=\nu_{0}$ allows to check, at the beginning of the diffusive zone $Q, \check{G}_{q c i}(\vec{R}, \varepsilon)=\check{G}_{Q}$.

${ }^{55}$ Y. Tserkovnyak, A. Brataas, G. E. W. Bauer, and B. I. Halperin, Rev. Mod. Phys. 77, 1375 (2005); A. Brataas, G. E. W. Bauer, and P. J. Kelly, Phys. Rep. 427, 157 (2006).

${ }^{56}$ The resemblance between our Eq. (58) and Eq. (49) of Ref. 57 is only formal because the $\hat{d}$ operator discussed by Yip for ballistic systems depends on the ballistic incidence angle. Furthermore, the angle average of Eq. (49) of Ref. 57 does not yield our Eq. (58), at least not straightforwardly.
${ }^{57}$ S.-K. Yip, J. Low Temp. Phys. 109, 547 (1997).

${ }^{58}$ In the definitions of $G_{\phi}^{L(R)}$ and $G_{\chi}^{L(R)}$ presented in Refs. 24 and 26 and Fig. 6 of Ref. 24, the indices $L(R)$ should be changed to $R(L)$. Then, the definitions of $G_{\phi}^{L(R)}$ and $G_{\chi}^{L(R)}$ given in these references are correct at zeroth order in $T_{n}$. This does not affect the results presented in these references.

${ }^{59}$ Quantum Noise in Mesoscopic Physics, edited by Yu. V. Nazarov (Kluwer, Dordrecht, 2003).

${ }^{60}$ K. Maki, in Superconductivity, edited by R. Parks (Marcel Dekker, New York, 1969).

${ }^{61}$ A. A. Abrikosov and L. P. Gor'kov, Sov. Phys. JETP 12, 1243 (1961).

${ }^{62}$ M. Houzet, V. Vinokur, and F. Pistolesi, Phys. Rev. B 72, 220506(R) (2005).

${ }^{63}$ W. Belzig, Ph.D. thesis, Karlsruhe University, 1999.

${ }^{64}$ P. G. de Gennes, Phys. Lett. 23, 10 (1966).

${ }^{65}$ L. N. Cooper, Phys. Rev. Lett. 6, 689 (1961).

${ }^{66}$ P. M. Tedrow, J. E. Tkaczyk, and A. Kumar, Phys. Rev. Lett. 56, 1746 (1986).

${ }^{67}$ R. Meservey and P. M. Tedrow, Phys. Rep. 238, 173 (1994).

${ }^{68}$ J. S. Moodera, X. Hao, G. A. Gibson, and R. Meservey, Phys. Rev. Lett. 61, 637 (1988); X. Hao, J. S. Moodera, and R. Meservey, Phys. Rev. B 42, 8235 (1990).

${ }^{69}$ X. Hao, J. S. Moodera, and R. Meservey, Phys. Rev. Lett. 67, 1342 (1991).

${ }^{70}$ J. A. X. Alexander, T. P. Orlando, D. Rainer, and P. M. Tedrow, Phys. Rev. B 31, 5811 (1985).

${ }^{71}$ Our numerical method is described in Section III of Ref. 25. We have used a Debye frequency $\Omega_{D}=601 k_{B} T$.

${ }^{72}$ S. Skalski, O. Betbeder-Matibet, and P. R. Weiss, Phys. Rev. 136, A1500 (1964).

${ }^{73}$ V. Ambegaokar and A. Griffin, Phys. Rev. 137, A1151 (1965).

${ }^{74}$ To check our prefactors, one can consider a $L(R)$ contact in the normal state and collinear regime (along $\vec{Z}$ ) with $L$ and $R$ in thermal equilibrium at potentials $\mu_{L}$ and $\mu_{R}$, respectively. In this case, one has $\check{K}_{L(R)}^{e, \sigma, \sigma}=2\left[1-2 f_{L(R)}^{e}(\varepsilon)\right]$ and $\check{K}_{L(R)}^{e, \sigma,-\sigma}=0$ for $\sigma$ $\in\{\uparrow, \downarrow\}$ with $f_{L(R)}^{e}(\varepsilon)$ the equilibrium distribution function for electrons at side $L(R)$ of the contact (see, e.g. Ref. 39). Combining Eq. (C4) and the expression $\left\langle I_{Q}\right\rangle=\int_{-\infty}^{\infty} d \varepsilon \operatorname{Tr}_{\sigma}\left\{\check{I}_{Q}^{K, e}(\varepsilon)\right\} / 8 e$ of the charge current, which derives from Eq. (67), one can recover exactly the Landauer-Büttiker expression (Ref. 50) $\left\langle I_{L(R)}\right\rangle$ $=\left(G_{T} / e\right) \int_{-\infty}^{\infty} d \varepsilon\left[f_{R}^{e}(\varepsilon)-f_{L}^{e}(\varepsilon)\right]$ for the charge current.

${ }^{75}$ M. J. M. de Jong and C. W. J. Beenakker, Phys. Rev. Lett. 74, 1657 (1995). 